\title{
Dynamic biomechanical model for assessing and monitoring robot-assisted upper-limb therapy
}

\author{
Hussein A. Abdullah, PhD, PEng;* Cole Tarry, BSc; Rahul Datta, BSc; Gauri S. Mittal, PhD, PEng; Mohamed \\ Abderrahim, PhD \\ School of Engineering, University of Guelph, Guelph, ON, Canada
}

\begin{abstract}
This article describes the design, validation, and application of a dynamic biomechanical model that assesses and monitors trajectory, position, orientation, force, and torque generated by upper-limb (UL) movement during robot-assisted therapy. The model consists of two links that represent the upper arm and forearm, with 5 degrees of freedom (DOF) for the shoulder and elbow joints. The model is a useful tool for enhancing the functionality of poststroke robot-assisted UL therapy. The individualized inertial segment parameters were based on anthropometric measurements. The model performed inverse dynamic analysis of UL movements to calculate reaction forces and moments acting about the 3-DOF shoulder and 2-DOF elbow joints. Real-time fused biofeedback of a 6-DOF force sensor and three-dimensional (3-D) pose sensors supported the model validation and application. The force sensor was mounted between the robot manipulator and the subject's wrist, while the 3-D pose sensors were fixed at specific positions on the subject's UL segments. The model input and output parameters were stored in the subject's database, which is part of the rehabilitation information system. We assigned 20 nondisabled subjects three different therapy exercises to test and validate the biomechanical model. We found that when the biomechanical model is taught an exercise, it can accurately predict a subject's actual UL joint angles and torques and confirm that the exercise is isolating the desired movement.
\end{abstract}

Key words: biomechanical model, inverse dynamics, kinematics, limb impairment, motion analysis, rehabilitation, robotassisted therapy, stroke, therapeutic robot, upper limb.

\section{INTRODUCTION}

Stroke is a leading cause of disability, particularly in older adults, and robot-assisted therapy techniques have important potential benefits. Stroke can cause both motor and sensory deficits that affect one or more limbs. Increasing evidence shows that active repetitive movement practice can have a profound effect on recovery of impaired motor function after stroke or brain injury [1-4]. However, long and intensive treatment is required. This treatment is costly and a major burden on the healthcare system and, as such, may result in inadequate duration of rehabilitation programs. One major issue is the need for a human attendant or caregiver to work with these patients. That repetitive, labor-intensive processes are easily and precisely handled by robots is widely accepted [5].

Abbreviations: $3-\mathrm{D}=$ three-dimensional, $\mathrm{ADL}=$ activities of daily living, $\mathrm{COM}=$ center of mass, $\mathrm{D}-\mathrm{H}=$ Denavit-Hartenberg, DOF = degrees of freedom, EMG = electromyography, MIT = Massachusetts Institute of Technology, PC = personal computer, $\mathrm{ROM}=$ range of motion, $\mathrm{UL}=$ upper limb.

*Address all correspondence to Hussein A. Abdullah, PhD, PEng; School of Engineering, University of Guelph, 50 Stone Road East, Guelph, Ontario, Canada N1G 2W1; 519824-4120, ext 53346; fax: 519-836-0227.

Email: habdulla@uoguelph.ca

DOI: 10.1682/JRRD.2006.03.0025 
During the 1990s, interest in rehabilitation robotics applications grew [6]. The first comprehensive work on robots in physiotherapy was done at the Rehabilitation Institute of Michigan, Detroit, Michigan, and employed a robotic arm [7]. The robot was programmed to perform five movement patterns, each consisting of eight points. Austin et al. developed another model for upper-limb (UL) rehabilitation using flexion/extension of the elbow in a horizontal plane [8]; however, the system controller was too position dependent. In a pilot study, Cozens used the principle of a robot-assisted, active single UL exercise [9]. The Massachusetts Institute of Technology (MIT) developed the MIT-Manus, which is a planar manipulator with 3 degrees of freedom (DOF) that was used in a series of clinical trials [10]. The clinical trial of the MIT-Manus for poststroke UL therapy found that manipulation of the impaired limb influenced recovery [11]. The Mirror Image Motion Enabler incorporated an industrial robot manipulator that applied forces to the paretic limb through a customized forearm splint [12]. The Assisted Rehabilitation and Measurement Guide, built by the Rehabilitation Institute of Chicago, Chicago, Illinois, has 3 controlled DOF and was designed to provide assistive therapy to patients with chronic hemiparesis [13].

Monitoring and assessing body kinematics, forces, and moments about the UL joints could improve the quality of robotic therapy. This monitoring and assessment could be achieved by a system that provides quantitative real-time feedback during the therapy process, followed by a mathematical assessment of the subject's recovery; such a system is currently unavailable.

The study of UL movement and motion analysis has attracted the attention of researchers and become an important tool in biomechanical modeling and clinical research. The UL is composed of three serial joints, the shoulder, the elbow, and the wrist. The connection of these joints by the upper arm and forearm allows a wide range of motion (ROM). Consequently, mechanical analysis of the UL requires information about the kinematics, forces, and moments generated at all three joints. While dynamic analysis of a rigid body presents no theoretical problem, the analysis of the UL is a complex task because of its anatomical features. Researchers looking for the best model to simulate UL mechanical properties have used various techniques and analytic methods. Technical and theoretical constraints make measurements of threedimensional (3-D) UL motion and forces difficult to collect and interpret. Modeling the wrist and elbow can be simplified with the use of two 1-DOF joints. The shoulder is a complex joint because it has two separate articulations, scapulothoracic and glenohumeral; a simple noninvasive way of locating the scapula is not available [14]. Since the early 1950s, several investigators have modeled the complex UL using various discrete masses, linear springs, and viscous dampers [15]. Previous mathematical analysis included the following assumptions: bones and tissues were considered rigid bodies, with their center of gravity fixed at a point, and joints were considered frictionless [16-18].

Optical systems with high resolution in space and time have been used to record 3-D kinematics of the UL [19]. A sensing garment that detects UL postures and movements has also been developed [20]. Analyses of UL movements are generally performed by measuring kinematic variables of the links and joints with accelerometers, electrogoniometers, or cameras [21-22]. Several experimental approaches have been developed to measure joint moments. Joint moments can be measured by torque transducers during isometric tasks or by torque motors during dynamics tasks $[19,23]$. All of these experimental approaches for measuring kinematics, forces, and moments restrict natural movement, require certain calibration and extra mounting fixtures, and can be invasive. Consequently, 3-D biomechanical models of the whole [16] or partial [24-27] UL have been developed. These models have used optimization methods to compute muscle forces and limb postures. Nieminen et al. used a 3-D model to predict maximum shoulder strength [27]. Raikova used mathematical analysis and the Denavit-Hartenberg (D-H) method to calculate position, muscle forces, and joint reactions for the UL [16]. Khalili and Zomlefer developed an approach to estimate the body segment parameters in a 2-D system for rehabilitation robotics [28]. Inverse dynamics can be used to calculate the UL reaction forces and moments at a joint. Kinematics represent the movement of the link-segment model, while inverse dynamics derive the kinetics responsible for that movement.

This article describes the design, validation, and application of a dynamic biomechanical model for monitoring and assessing a subject's UL trajectory, position, and orientation during robot-assisted therapy. The model also computes the 5-DOF joint reaction forces and torques generated when the therapeutic robot manipulates the UL. Simultaneous monitoring of real-time fused biofeedback with a 6-DOF force sensor and 3-D pose sensors validates the therapeutic task. Determining the 3-D mechanical 
properties of the UL during functional movement is also possible. This biomechanical model has been developed in conjunction with a novel pilot version of a therapeutic robot. We studied a group of 20 nondisabled subjects to validate the model. Subjects participated in three robotassisted passive therapy exercises: elbow, shoulder, and combined (elbow-shoulder). The model results and performance were analyzed.

\section{METHODS}

\section{Biomechanical Model Development}

We present a noninvasive, flexible, and reliable method to assess and monitor biomechanical quantities by inverse dynamics and supported biosensors. This method can improve the quality and sensitivity of clinical analysis in poststroke robot-assisted therapy. The first therapeutic robot prototype we developed employed electromyography (EMG) measurements as biofeedback for assessing recovery over time [29]. However, after implementing the surface electrode EMG, we found that capturing the same muscles in subsequent training sessions was difficult. We could overcome this difficulty using needle or fine-wire electrodes; however, physiotherapists are not qualified to perform such procedures and they would drastically increase cost and time requirements. Therefore, we investigated improving the model-assessment accuracy by integrating two different biofeedback measurements, force and position. In the current system, we used a 6-DOF force sensor to measure the force and moment around three separate axes: $x, y$, and $z$. The force sensor was mounted between the robot manipulator and the subject's wrist. The system was equipped with 3-D pose sensors that were fixed at specific positions on the subject's upper arm and forearm. Real-time fused biofeedback of the force sensor and 3-D pose sensors was recorded by the system, and kinematics, posture, and motion functionality measures of the therapeutic robot were calculated. This biomechanical model determined orientation, position, angular and linear velocities, and the amount of force and moment about the UL joints.

Our approach used inverse dynamics theory, an iterative Newton-Euler dynamics algorithm [30], and biofeedback collected from the force and 3-D pose sensors to calculate actual UL postures, velocities, forces, and joint torques.
The biomechanical model we developed has the following capabilities:

- It completely implements UL kinematic and kinetic models.

- It was developed based on the anthropometry of the target population.

- It monitors and stores data for each modeled DOF: position; orientation; linear and angular velocity; and acceleration, force, and torque.

- It maintains a subject database for every session of the exercise.

- It compares the exercise trajectory and related parameters taught to it by the therapist with the "Teach and Repeat" model with the actual trajectory, velocity, and forces achieved by the subject.

\section{Modeling Assumption}

When modeling UL movement, we must consider the limitations of patients who require poststroke rehabilitation. Modeling of the shoulder (socket joint) can be overly complex with respect to the simple motions that patients must relearn to be able to perform activities of daily living (ADL). The goal of this model was to acquire and monitor information about ROM and joint strength and determine a patient's ability to accomplish basic ADL. We did not need to model the limb exactly, and therefore, the calculated DOF of the limb were reduced. The number of DOF of an articulated body is the number of joint angles necessary to specify the state of the structure, which is a series of rigid links connected at joints. The model was also based on the following assumptions: the joints of the UL are frictionless pin-joints, the UL segments are rigid with mass concentrated at their centers of mass (COMs), and air friction is insignificant. The effect of these assumptions was minimal on the model output, because the main focus of the system was comparison of a subject's progress relative to a previous exercise performance. All performance analysis of the different exercises was based on the same assumptions.

\section{Kinematics Model}

A good algorithm for solving the rehabilitation kinematics problem must be both fast and interactive. It should also be able to find a sensible solution that makes the UL posture acceptable. Usually, the kinematics function is highly nonlinear and rapidly becomes more and more complex as the number of links increases. Thus, the inversion of the function could become impossible to perform 
analytically. Consequently, we proposed to model the whole UL as seven 1-DOF revolute joints. The kinematics model of the UL was created to properly propagate joint and limb dynamics in the algorithm. The revolute joints modeled the shoulder, elbow, and wrist, which are connected by two links that represent the upper arm and forearm. The model represented the shoulder as a 3-DOF spherical joint (the glenohumeral joint) and the elbow as a 2-DOF joint (Figure 1). The wrist was not included in the model because it was considered a fixed joint and the point at which all forces and moments were read for the robotic therapy system. The wrist represented the rigid contact point between the robot manipulator, the force sensor, and the subject's arm. We were not concerned with the development of a complex biomechanical model of the UL, but rather with work-space analysis and interactive posturing applications that could enhance robot-assisted therapy.

The 5-DOF model of the UL is shown in Figure 2 and consists of 7 frames or joint DOF. The base of the model, frame 0 , is the center of the shoulder joint. Frames 1-6 correspond to the modeled joint DOF of the UL joints. Frames 1-3 represent the 3-DOF of the shoulder. Frame 1

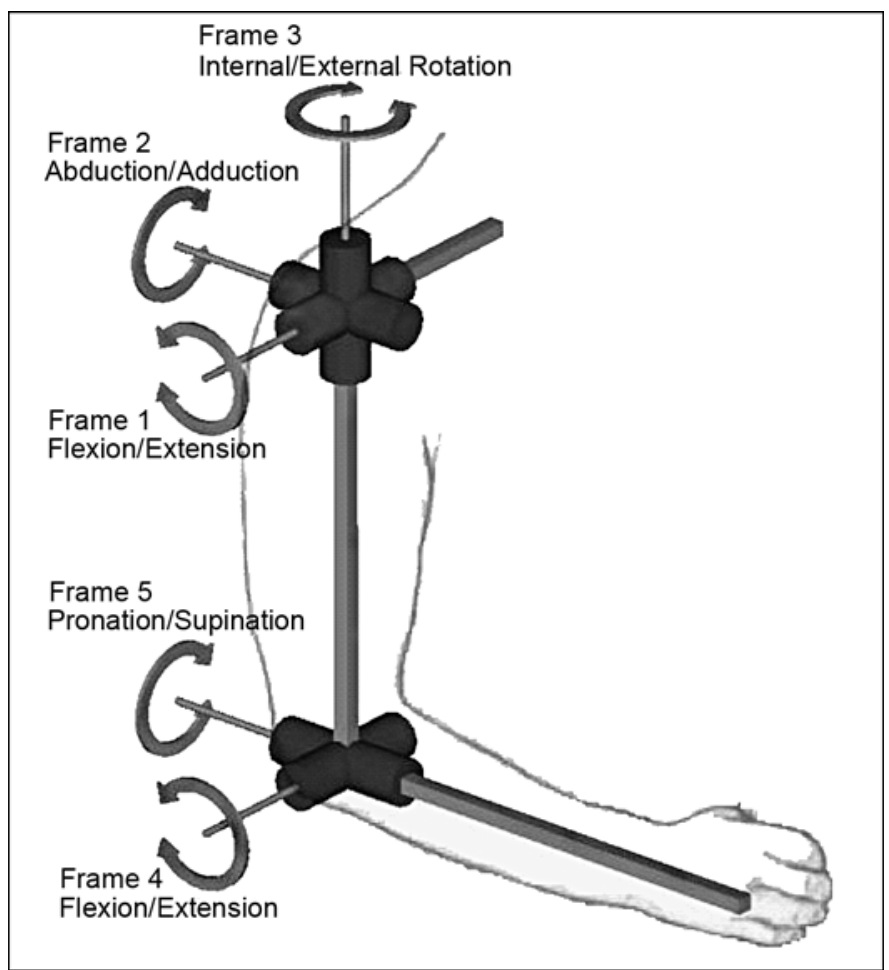

Figure 1.

Kinematics diagram of proposed biomechanical model showing frame designation for joints' modeled degrees of freedom. allows flexion/extension of the upper arm, frame 2 abduction/adduction of the upper arm, and frame 3 the internal/ external rotation. Frames $4-5$ represent the 2-DOF of the elbow; frame 4 allows flexion/extension and frame 5 pronation/supination of the forearm. These designations are detailed in Table $\mathbf{1}$ and can be used to model static or dynamic functions of the UL in any direction.

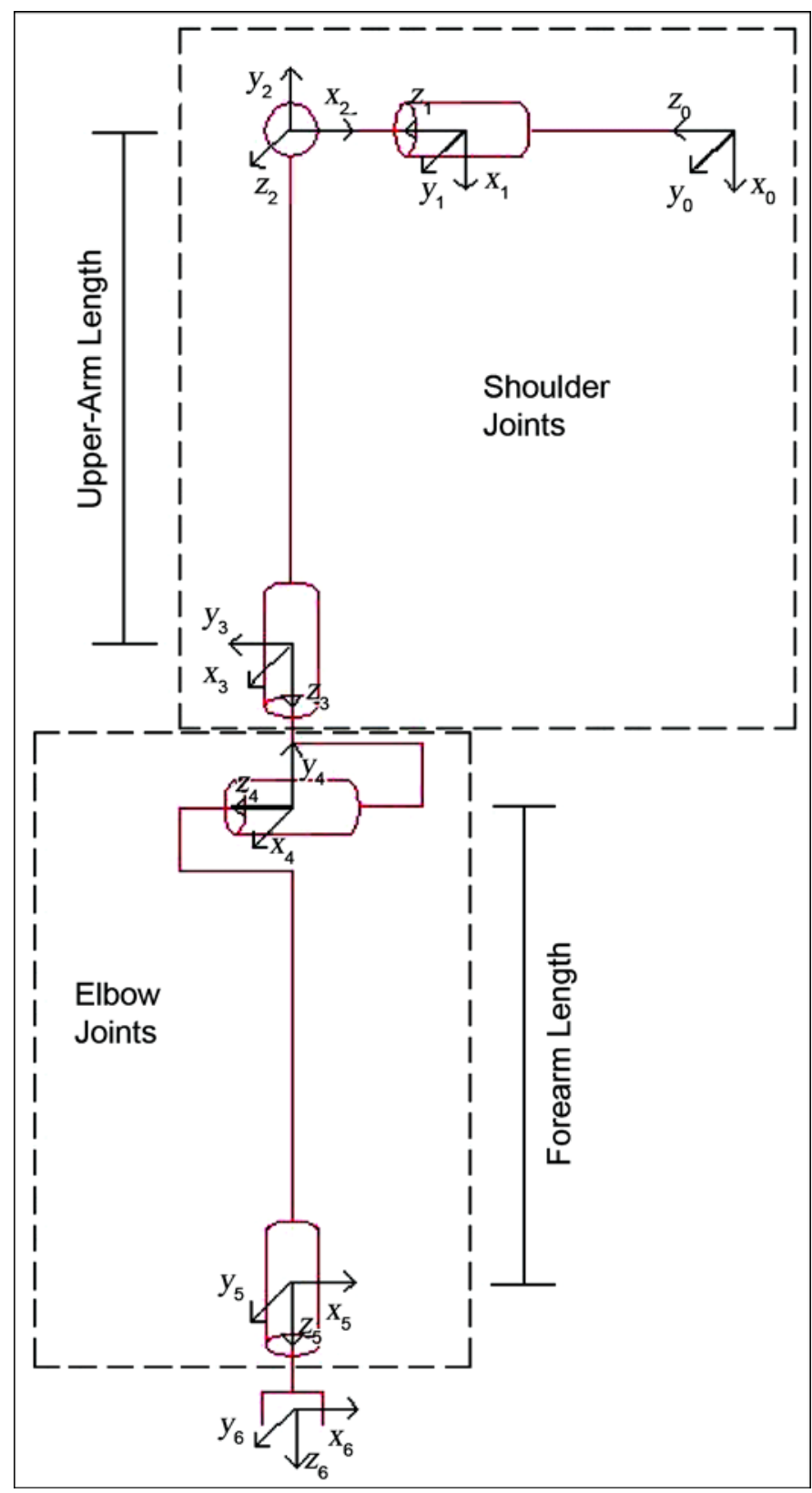

Figure 2.

Local coordinate systems defined for upper-limb model. Numbers refer to joints' modeled degrees of freedom. 
Once the UL model was created, we extracted the modified D-H parameters [31]. We developed subsequent transformation matrices from the D-H table to reference each frame of the model to the next frame (Table 2). These parameters are defined as part of Equation 2. The matrices were used for velocity, acceleration, and force propagation equations within the Newton-Euler algorithm.

After the model's segment and link structure was created, we needed to define the known frame of the local coordinate system for each respective joints' DOF to enable the parametric description of its state. Such description was fully achieved with a transfer matrix that related the joint DOF relative frame to its reference frame (frame 0). Each frame had its own origin and 3-D axes $(x, y$, and $z)$. The rotation was assumed to be about the $z$-axis of each frame,

$$
R_{z}(\theta)=\left[\begin{array}{ccc}
\cos (\theta) & -\sin (\theta) & 0 \\
\sin (\theta) & \cos (\theta) & 0 \\
0 & 0 & 1
\end{array}\right],
$$

Table 1.

Frame or joint degrees of freedom representations for upper-limb model.

\begin{tabular}{ccl}
\hline Frame & Location & \multicolumn{1}{c}{ Functionality } \\
\hline 0 & $\begin{array}{c}\text { Shoulder } \\
\text { Center }\end{array}$ & Base of model coordinate system. \\
& Shoulder & Flexion/extension upper arm. \\
2 & Shoulder & Abduction/adduction upper arm. \\
3 & Shoulder & Internal/external rotation upper arm. \\
4 & Elbow & Flexion/extension forearm. \\
5 & Elbow & Pronation/supination wrist and forearm. \\
6 & Wrist & Wrist location. \\
\hline \hline
\end{tabular}

Table 2.

Denavit-Hartenberg table configuration for upper-limb model. $a_{i-1}$ was distance from $z_{i-1}$ to $z_{i}$ along $x_{i}$ axis; it is a model dependent parameter and is always zero, because all $z$-axes were coincident or perpendicular.

\begin{tabular}{cccc}
\hline Frame $\boldsymbol{i}$ & $\boldsymbol{i}-\mathbf{1}$ & $\boldsymbol{d}_{\boldsymbol{i}}$ & $\theta_{\boldsymbol{i}}$ \\
\hline 1 & 0 & 0 & $\theta_{1}$ \\
2 & -1.57 & 0 & $\theta_{2}$ \\
3 & 1.57 & Upper-arm length & $\theta_{3}$ \\
4 & -1.57 & 0 & $\theta_{4}$ \\
5 & 1.57 & Forearm length & $\theta_{5}$ \\
6 & 0 & 0 & 0 \\
\hline$\theta_{i}=$ rotation angle for frame $i$. & \\
$d_{i}$ was distance between $x_{i-1}$ and $x_{i}$ along $z_{i}$ axis. \\
$i-1$ was rotation angle between z-axis of joint degree of freedom (DOF) $i-1$ \\
and joint DOF $i$.
\end{tabular}

where $R_{z}=$ rotational matrix about the $z$-axis, $\theta=$ angle of rotation about the $z$-axis, cos $=$ cosine, and $\sin =$ sine.

Seven local coordinate systems were defined for frames $i=0,1,2, \ldots 6$ (Figure 2 ), and the $(4 \times 4)$ homogeneous transformation matrix that described the rotations and translations for aligning frame $i$ to frame $i-1$ was

$$
{ }_{i}^{i-1} T=\left[\begin{array}{lccc}
\cos \theta_{i} & -\sin \theta_{i} & 0 & a_{i-1} \\
\sin \theta_{i} \cos \alpha_{i-1} & \cos \theta_{i} \cos \alpha_{i-1} & -\sin \alpha_{i-1} & -\sin \alpha_{i-1} d_{i} \\
\sin \theta_{i} \sin \alpha_{i-1} & \cos \theta_{i} \sin \alpha_{i-1} & \cos \alpha_{i-1} & \cos \alpha_{i-1} d_{i} \\
0 & 0 & 0 & 1
\end{array}\right],(
$$

where ${ }^{i-1}{ }_{i} \mathrm{~T}$ was the transformation matrix that converted the coordinates in frame $i$ to those in frame $i-1, \theta_{i}$ was the rotation angle for joint DOF $i, d_{i}$ was the distance between $x_{i-1}$ and $x_{i}$ along the $z_{i}$ axis, and $\alpha_{i-1}$ was the rotation angle between the $z$-axis of joint DOF $i-1$ and joint DOF $i$ [30]. The distance from $z_{i-1}$ to $z_{i}$ along the $x_{i}$ axis was $a_{i-1}$, which is a model dependent parameter and for our model was always zero, because all the z-axes were coincident or perpendicular (Figures 1-2). Detailed information about the modified $\mathrm{D}-\mathrm{H}$ parameters can be found in Craig [30]. The model calculated joint velocities, acceleration, and forces, which were required to determine joint torques on the links when dynamics of the system were investigated.

\section{Position and Orientation}

The direct kinematics problem consisted of finding the position and orientation of the upper arm and forearm with respect to a fixed-reference coordinate system. The model must track and measure the position and orientation of the UL segments and joint angles in real time as the therapy exercise is performed. The inverse kinematics problem involved determining the joint variables given the position and the orientation of the UL segment with respect to the reference coordinate system. The model calculated the Cartesian arm position and orientation taught to it by the therapist and correlated them with the feedback from the 3-D pose sensors mounted on the subject's UL as therapy exercise was performed.

When investigating which sensors to use and where to mount them on the subject's UL, we must allow the patient full flexibility, while being accurate and safe. We acquired 3-D pose sensors and mounted them to a custom-made fixture and arm strip that attached to a subject's UL. The model needed to track UL position and orientation accurately and in real time. 
Two 3-D pose sensors were used to track the UL (Figure 3); one located at the interface between the robot and the subject's wrist and the other attached to the upper arm centered over the elbow joint. Each sensor was capable of measuring its location $(x, y$, and $z)$ in space as well as its orientation (azimuth, elevation, and roll) with respect to a stationary base point. These data were used as the five joint angles of the UL model.

\section{Masses and Forces}

Calculating the reaction forces and moments at the UL joints required modeling of the upper arm and forearm. Several parameters were required for the calculations, such as the limb's length and the subject's body weight and height; these data formed the model's input parameters. Other parameters such as COM, segment weight, and radius of gyration were calculated based on the ratios of anthropometric data [32-33] and tables available in

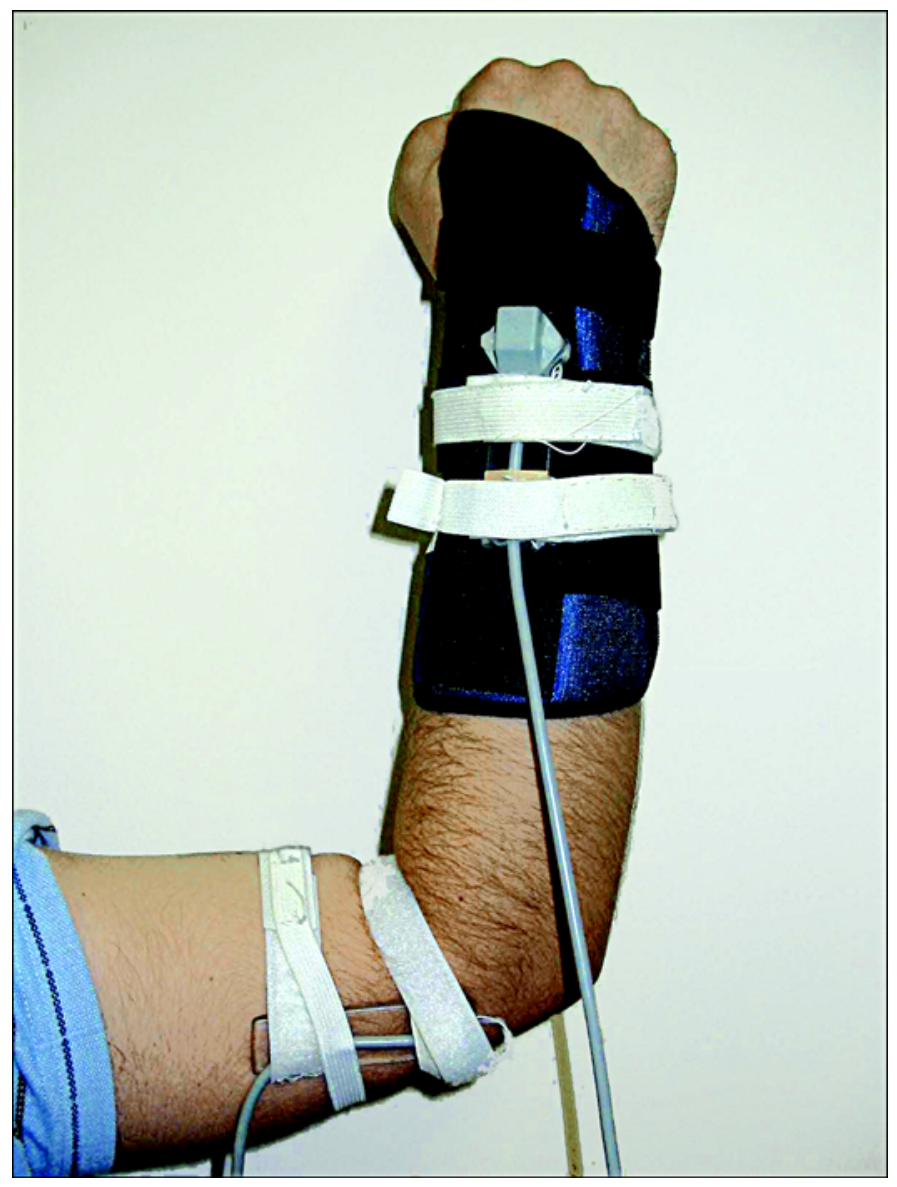

Figure 3.

Three-dimensional pose sensors attached to subject's wrist and elbow. the literature. This will allow us to easily modify the model to accommodate the largest and smallest UL of the target population. We calculated the moments of inertia for UL segments using standard mathematical equations for homogeneous bodies [30]. In our model, the variation between an individual's actual dimensions and those calculated from anthropometric data did not have a negative effect on the therapy evaluation because an individual's improvement was measured by comparing current with previous performance and both calculations contained the same model parameters. Any change in the model output was caused by a change in the individual's performance, since the values relating to the physical dimensions were kept constant.

The model can estimate the joint torques required for a person to move the limb unassisted. However, in rehabilitative therapy, the patient's motions are assisted and resisted in an attempt to improve motor function, including ROM and strength. To measure the improvement of the patient, we needed to measure the forces resulting from the system.

During the therapy exercise, the biomechanical model sensed the forces and torques at the human-robot interface through the 6-DOF force transducer attached to the subject's wrist. This provided the net effect of the forces in the system; those applied by the subject and those generated by the robotic manipulator. The model used the inverse dynamics and an iterative Newton-Euler algorithm computation to calculate the reaction forces at the UL joints and record them as biofeedback in real time. Physiotherapists could then update the therapy regime according to this feedback. Therefore, this system could enhance a subject's comfort and improve the productivity, quality, and safety of the therapy.

Equations 3-7 calculated iteratively outward from the base of the manipulator, or in this case the subject's shoulder, to acquire the velocity and acceleration of each frame as explained in Craig [30]

$$
\begin{gathered}
{ }^{i+1} \omega_{i+1}={ }^{i+1} R^{i} \omega_{i}+\dot{\theta}_{i+1}{ }^{i+1} \hat{Z}_{i+1}, \\
{ }^{i+1} v_{i+1}={ }_{i}^{i+1} R\left({ }^{i} v_{i}+{ }^{i} \omega_{i} \times{ }^{i} P_{i+1}\right), \\
{ }^{i+1} \dot{\omega}_{i+1}={ }^{i+1} R^{i} \dot{\omega}_{i}+{ }_{i}^{i+1} R^{i} \omega_{i} \times \dot{\theta}_{i+1}{ }^{i+1} \hat{Z}_{i+1}+\ddot{\theta}_{i+1}{ }^{i+1} \hat{Z}_{i+1}, \\
{ }^{i+1} \dot{v}_{i+1}={ }_{i}^{i+1} R\left({ }^{i} \dot{\omega}_{i} \times{ }^{i} P_{i+1}+{ }^{i} \omega_{i} \times\left({ }^{i} \omega_{i} \times{ }^{i} P_{i+1}\right)+{ }^{i} \dot{v}_{i}\right) \text {, and } \\
{ }^{i+1} \dot{v}_{C i+1}={ }^{i+1} \dot{\omega}_{i+1} \times{ }^{i+1} P_{C i+1}+{ }^{i+1} \omega_{i+1} \times\left({ }^{i+1} \omega_{i+1} \times{ }^{i+1} P_{C i+1}\right)+{ }^{i+1} \dot{v}_{i+1},
\end{gathered}
$$


where $\omega$ and $\dot{\omega}$ were the angular velocity and acceleration vectors respectively, $v$ and $\dot{v}$ were the linear velocity and acceleration vectors, respectively, $R$ was the rotation matrix (Equation 1), $\theta$ was the angular displacement, $P$ was the position vector of the frame, $P_{C}$ was the position vector of the link COM, and $\dot{v}_{C}$ was the acceleration vector of the link COM.

Equations 8-9 calculated the force $(F)$ and torque $(N)$ about the COM of each link [30] by

$$
\begin{gathered}
{ }^{i+1} F_{i+1}=m_{i+1}{ }^{i+1} \dot{v}_{C i+1} \text { and } \\
{ }^{i+1} N_{i+1}={ }^{C_{i+1}} I_{i+1}{ }^{i+1} \dot{\omega}_{i+1}+{ }^{i+1} \omega_{i+1} \times{ }^{C^{i+1}} I_{i+1}{ }^{i+1} \omega_{i+1}
\end{gathered}
$$

where $m=$ mass of the link and $I=$ inertia tensor of the link. Equations 10-11 calculated iteratively inward from the robot manipulator or, in this case, the subject's wrist. This ensured that the force and torque of each joint DOF included those that followed it [30]:

$$
\begin{gathered}
{ }^{i} f_{i}={ }_{i+1}^{i} R^{i+1} f_{i+1}+{ }^{i} F_{i} \text { and } \\
{ }^{i} n_{i}={ }^{i} N_{i}+{ }_{i+1}^{i} R^{i+1} n_{i+1}+{ }^{i} P_{C i} \times{ }^{i} F_{i}+{ }^{i} P_{i+1} \times{ }_{i+1}^{i} R^{i+1} f_{i+1},
\end{gathered}
$$

where $f_{i}$ was the force exerted on joint DOF $i$ by joint DOF $i-1$, and $n_{i}$ was the torque exerted on joint DOF $i$ by joint DOF $i-1$.

By comparing the subject's requirements with the actual forces recorded by the system, we can determine how much motion is the result of the subject's abilities and to what degree the robot is assisting the motion.

The objective is to isolate the forces at each of the modeled joints. This allows for a quantitative measure of the performance at each joint of the UL (whether active or passive) that can be compared with subsequent repetitions and sessions of each exercise, which would allow a subject's performance to be compared with another subject's progress as well as his or her own progress.

\section{Implementation of Biomechanical Upper-Limb Model}

The mathematical model was implemented in a Microsoft Excel 2003 spreadsheet (Microsoft, Corp, Redwood, California), which allowed all stages of the forward and inverse kinematics as well as the dynamics model to be visible to the user. The first phase of the Newton-Euler algorithm calculated the angular and linear velocities and accelerations for each frame in an outward fashion from frame 0 to 6 . The second phase of the Newton-Euler algo- rithm was implemented by iterative calculation of the forces and moments for each frame in a backward fashion. The inputs to this phase were the kinematics data (joint angles, velocities, and accelerations), which were obtained by the kinematics model explained in the "Kinematics Model" section (p. 45). Additional inputs to the model were the external force measured by the force sensor and the exercise trajectory data measured by the 3-D pose sensors. We estimated parameters such as mass, length, COM, and moments of inertia of the subject's UL using the anthropometric data tables integrated in the model.

The input parameters (length of upper arm and forearm, body weight, and sex) required by the model were entered through a graphical user interface (Figure 4). Based on these inputs, parameters such as COM and values of the angle moved by each frame during the UL movement were calculated. The UL segment's position, trajectory, and orientation were measured by the 3-D pose sensors and recorded through the robot-computer interface. Forces and torques at frame 6 were measured by the force sensor and recorded through the robot-computer interface. The outputs of the model were the reaction forces and torques acting on the UL at the elbow and shoulder joints.

We can conclude that the biomechanical model has the following benefits:

1. It provides real-time feedback during the therapy process.

2. It monitors, records, fuses, and stores the force sensor and 3-D pose sensor outputs.

3. It provides quantitative information not currently available to therapists (ROM, force profile, movement efficiency, and velocity).

4. It is a noninvasive method that does not restrict movement, and it is easily mounted on the limb.

Moreover, after performing a clinical trial, we expect that the model will improve therapy quality and reduce necessary therapy time. However, this conclusion requires further experimental data to be verified.

\section{Experimental Setup}

To test its validity, we used the model in conjunction with a pilot version of a therapeutic robotic developed previously. The system consists of a CRS-255 robotic manipulator (CRS Plus, Burlington, Ontario, Canada) capable of 5DOF movement, a 6-DOF force transducer (JR3, Inc, Woodland, California) sampled at $100 \mathrm{~Hz}$, and 3-D pose sensors capable of measuring location and orientation 


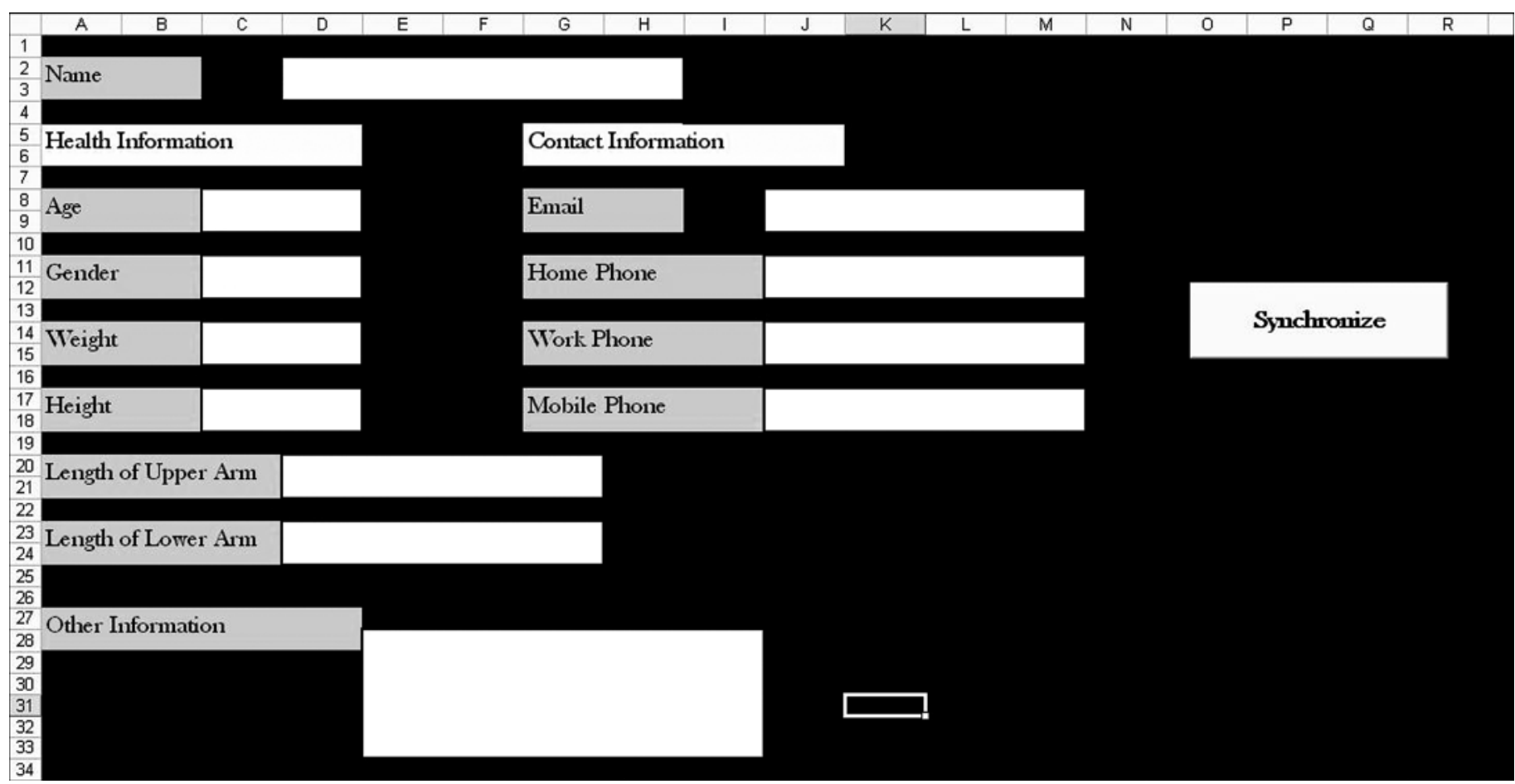

Figure 4.

Registration form for quantitative assessment tool. Graphical user interface for entering input parameters.

(Patriot $^{\mathrm{TM}}$, Polhemus, Colchester, Vermont) sampled at $60 \mathrm{~Hz}$. A personal computer (PC) was used as the main controller, and all the sensor outputs were linked to the PC through a universal serial bus data acquisition card. With the 3-D pose sensors attached to the subject's UL at the wrist and elbow joints, the therapist can maneuver the UL through an exercise while the system tracks and learns the movement in 3-D space. The robot can then assist the subject's movement through the specific exercises developed by the therapist. This unique "Teach and Repeat" function allows therapists to teach the therapeutic robot an exercise in a manner that is natural to their training. The robotic system can learn any therapy exercise (trajectory, velocity, and orientation) offline, within the subject's work space. The therapist can move the UL through the recommended exercise and the system can capture and automatically record the exercise information. The robot has a precision of \pm 0.05 $\mathrm{mm}$; with preprogrammed exercises, the movement path of the robot will be essentially identical. Any difference between the robot's location and the subject's wrist is related to a slight offset based on the width of the subject's UL and its orientation due to the free joints that attach the wrist to the force sensor. The PC interface program has an
OpenGL graphic window (SGI, Sunnyvale, California) (Figure 5) that allows the therapist to view a virtual model of the subject's UL and the recorded exercise trajectory.

Reviewing the recorded exercise while the robot manipulator is offline adds an extra layer of safety for both the subject and the robot system. The upper left of Figure 5 shows the simulation model of the UL, while the lower left shows the therapy exercise. The right of Figure 5 shows the other features of the "Teach and Repeat" learning model. A diagram of the experimental setup is shown in Figure 6.

We assigned 20 nondisabled subjects (Table 3) three different treatment exercises to test and validate the biomechanical model. Experimental subjects had the following characteristics:

- Age: range 19 to 58 yr.

- Sex: 5 females and 15 males.

- Height: range 1.58 to $1.86 \mathrm{~m}$.

- Weight: range 49 to $89 \mathrm{~kg}$.

We taught the robot three exercises using the "Teach and Repeat” learning feature. One exercise isolated flexion/ extension of the elbow joint, another isolated the 3-DOF of 


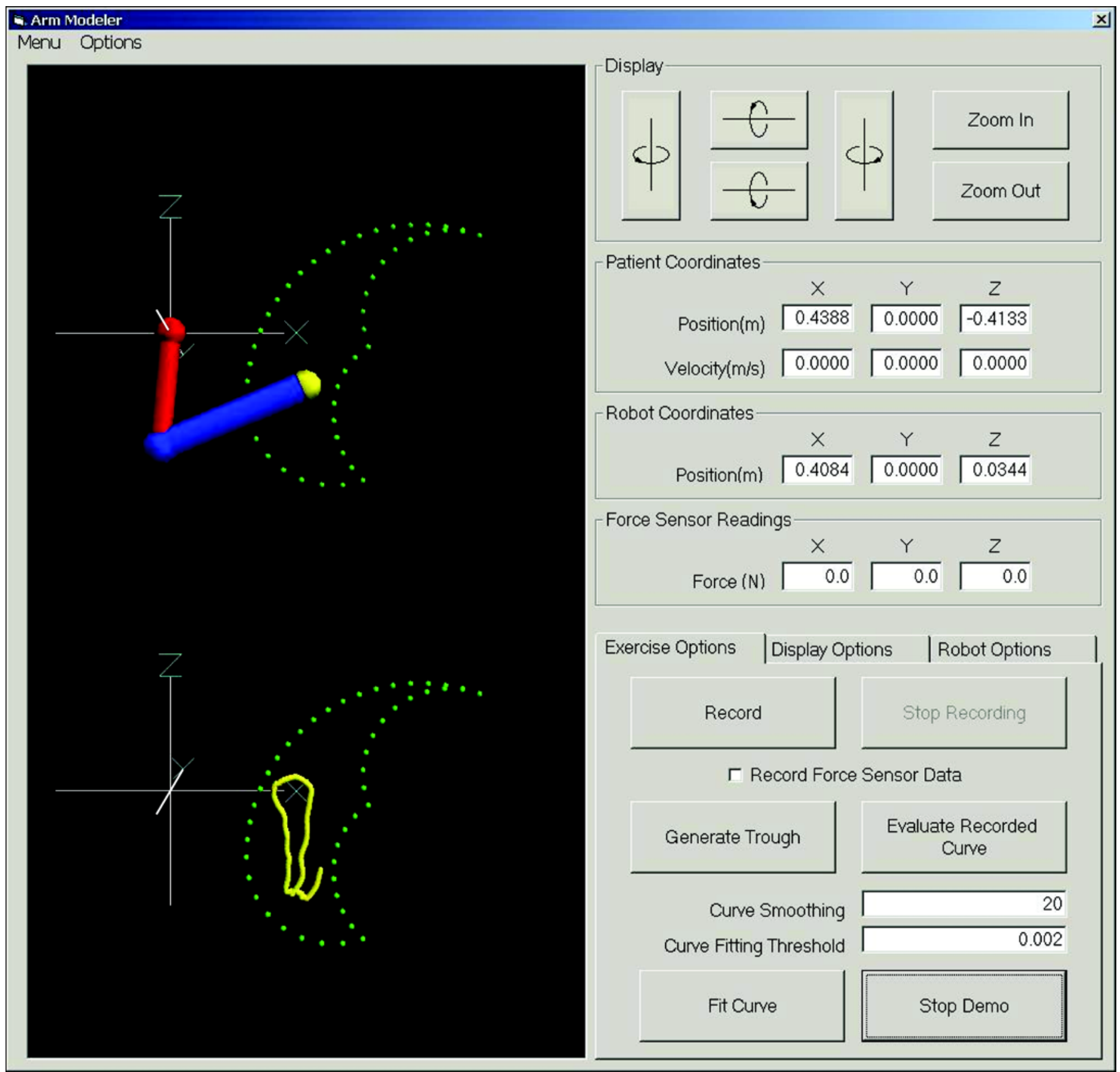

Figure 5.

“Teach and Repeat” module graphical user interface.

the shoulder, and the third combined the motion of the shoulder and the elbow. A custom-made splint was used to attach the subject's wrist to the force sensor that was mounted directly to the robot. The experimental protocol was approved by the University of Guelph Research Ethics Board.

\section{Procedures and Exercises}

Each subject went through a session of the three different exercises with each exercise pattern repeated 4 times and then 12 times in passive mode. A rest period of 10 min was allowed between each exercise. In passive mode, the subjects were instructed to relax and let the 
robot move the UL. The subject's wrist velocity was held constant at $5.5 \mathrm{~cm} / \mathrm{s}$. During the exercise, we recorded data pertaining to force and position for the subject's UL using a force sensor and 3-D pose sensors and a data acquisition system.

Elbow Exercise. This exercise generated reaction forces and moments at the elbow joint.

- Step I: The subject's UL was at his or her side with the elbow bent to $90^{\circ}$.

- Step II: The robot moved in a trajectory such that elbow flexion increased.

- Step III: The robot moved back to the original position by extending the elbow again.

Figure 7 illustrates the UL movement during the elbow exercise.

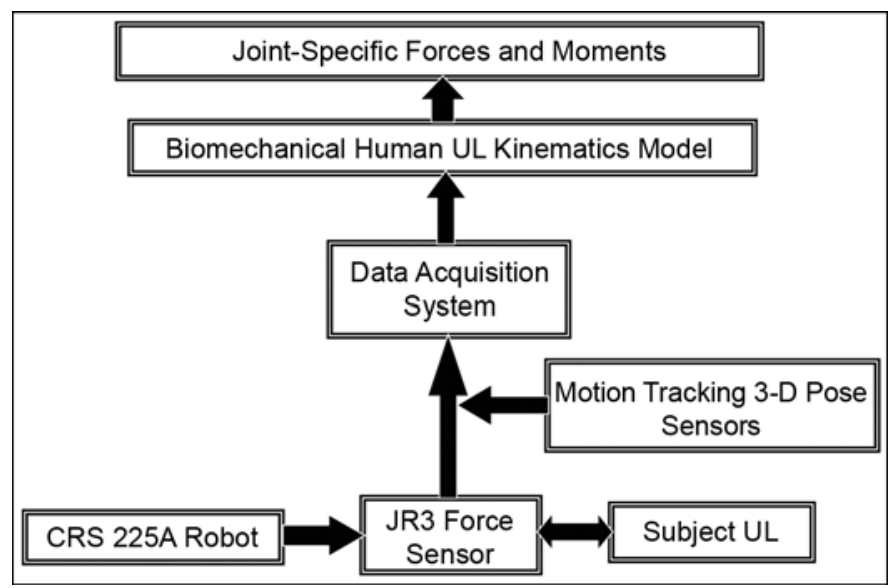

\section{Figure 6.}

Flow diagram of experimental setup. CRS-255A robotic manipulator (CRS Plus, Burlington, Ontario, Canada) capable of 5-DOF movement. JR3 6-DOF force sensor (JR3 Inc, Woodland, California). 3-D = threedimensional, $\mathrm{DOF}=$ degrees of freedom, $\mathrm{UL}=$ upper limb.

Table 3.

Subject data for evaluation of model.

\begin{tabular}{lc}
\hline \multicolumn{1}{c}{ Variable } & Mean \pm SD \\
\hline Age (yr) & $31.1 \pm 10.0$ \\
Body Weight $(\mathrm{kg})$ & $67.4 \pm 11.1$ \\
Upper-Arm Length (m) & $0.30 \pm 0.05$ \\
Forearm Length (m) & $0.39 \pm 0.04$ \\
Upper-Arm Mass (kg) & $1.89 \pm 0.30$ \\
Forearm Mass (kg) & $1.48 \pm 0.24$ \\
\hline SD = standard deviation. & \\
\hline \hline
\end{tabular}

Shoulder Exercise. This exercise generated reaction forces and moments at the shoulder joint.

- Step I: The subject's UL was fully extended forward and to the right of the shoulder.

- Step II: The robot moved along a trajectory that resembles a "V." This moved the subject's hand down to waist level and directly centered in front. The robot then moved the UL up and to the left to complete the "V." The UL was at full extension through out the entire exercise.

- Step III: The robot then moved back along the same trajectory, returning the subject's UL back to its start position.

Figure 8 illustrates the UL movement during the shoulder exercise.

Combined Exercise. The combined exercise involved efforts both at the elbow and the shoulder.

- Step I: The subject's UL was initially situated at his or her side with the elbow bent to $90^{\circ}$.

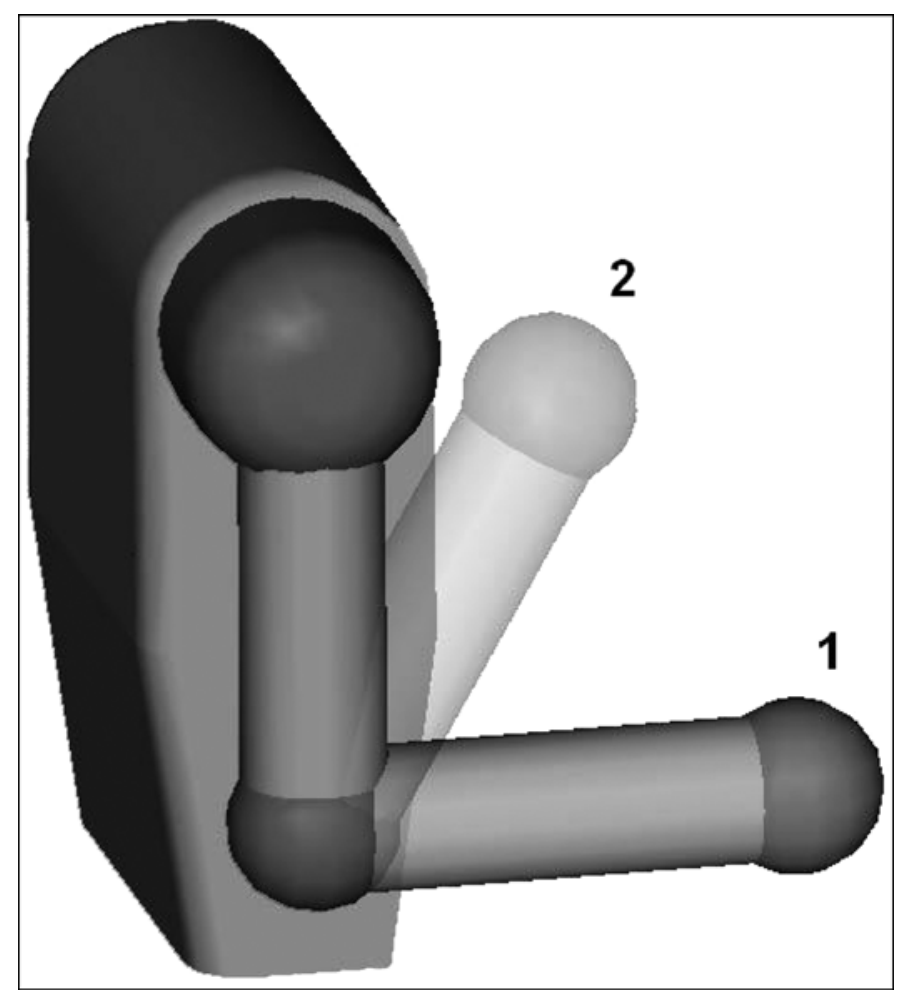

Figure 7.

Elbow exercise that generates forces and moments at elbow joint. $1=$ steps I and III, 2 = step II. 


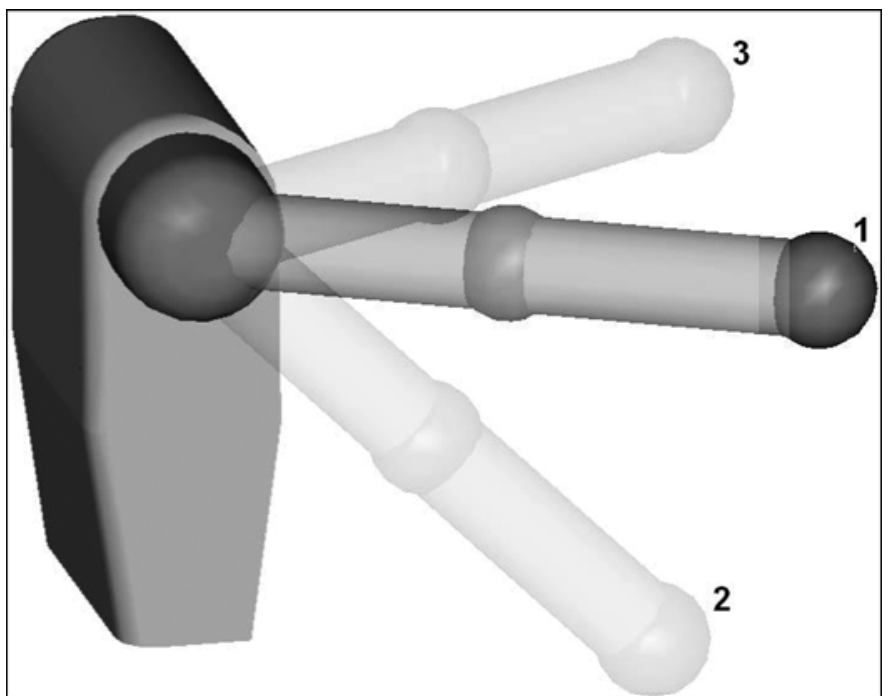

Figure 8.

Shoulder exercise that generates forces and moments at shoulder joint. 1 = step I, 2 = step II, 3 = step III.

- Step II: The robot moved in a trajectory such that the subject's hand moved across the front of his or her body and ended in near full extension of the UL, with the subject's hand located close to his or her opposite shoulder.

- Step III: The robot then moved back along the same trajectory, returning the subject's UL to its starting position.

Figure 9 illustrates the UL movement during the combined exercise.

\section{EXPERIMENTAL RESULTS}

The results are based on the experimental investigation on 20 nondisabled subjects that we conducted to evaluate the model's performance. Figure $\mathbf{1 0}$ shows the experimental and model-assessed results of an elbow exercise on one subject. The left-hand column presents the 5-DOF joint angles of the UL, detailed in Figure 2, as recorded by the 3-D pose sensors. The right column presents the model-assessed torque on the UL joints caused by the motion during the elbow exercise and derived from the transformation of the force-sensor data. We can see from the plots that movement was isolated to joint DOF 4 (flexion/extension of the forearm), as was the intention of the exercise. These data show that the largest angle

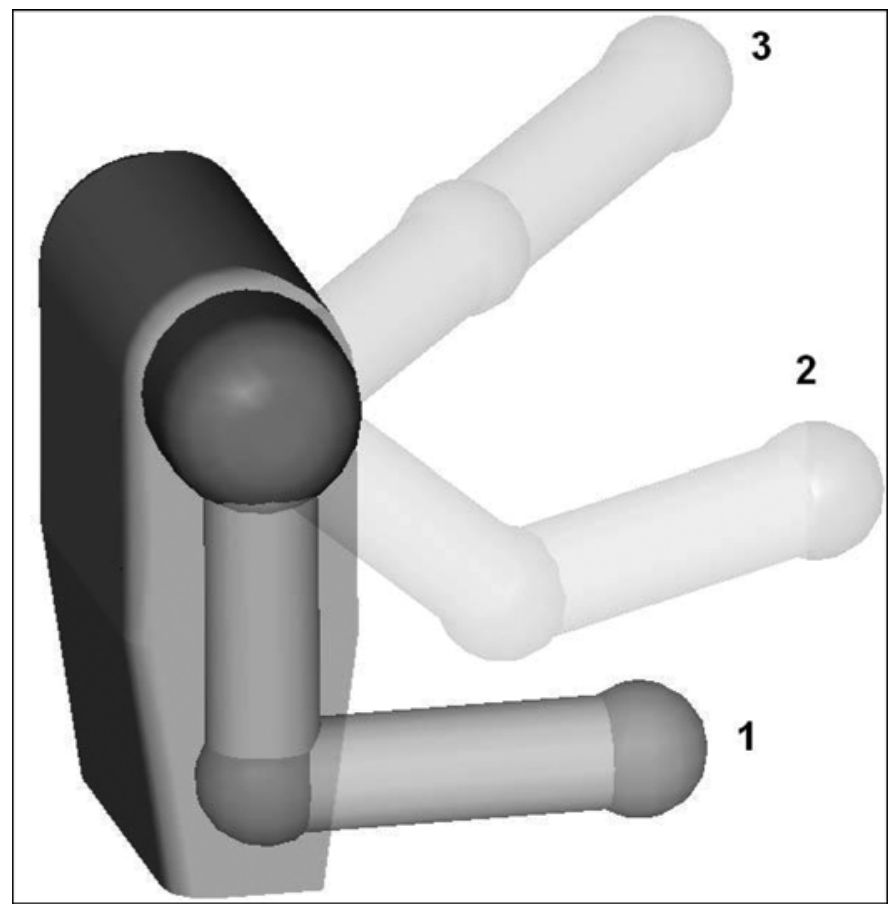

Figure 9.

Combined exercise that involves forces at both elbow and shoulder joints. 1 = step I, 2 = step II, 3 = step III.

(movement) occurred about the $z$-axis of joint DOF 4 . The torque on the joint DOF was propagated from the robotlimb contact point backward using the biomechanical model. The torque on joint DOF 1 , which ran relatively parallel to joint DOF 4 in this exercise, was the largest because it needed to compensate for the reaction of the torque on joint DOF 4 and the weight of the UL. The torque and movement on the other joints were minimal and relatively constant as they rotated perpendicular to the axis of rotation in this experiment. The elbow exercise was designed to isolate the flexion/extension of the forearm at the elbow joint. That it did so is obvious from the results generated by the experimental test of the model in Figure 10; therefore, the model was responsive to the actual elbow exercise.

The results from the shoulder exercise are shown in Figure 11. The shoulder exercise involved all the joints except joint DOF 5, which represented pronation/supination of the wrist and forearm. We can conclude that the maximum displacement and torque occurred at joint DOF 1. Again, these results confirm that when the biomechanical model is taught an exercise, it can accurately predict a subject's actual UL joint angles and torques. 

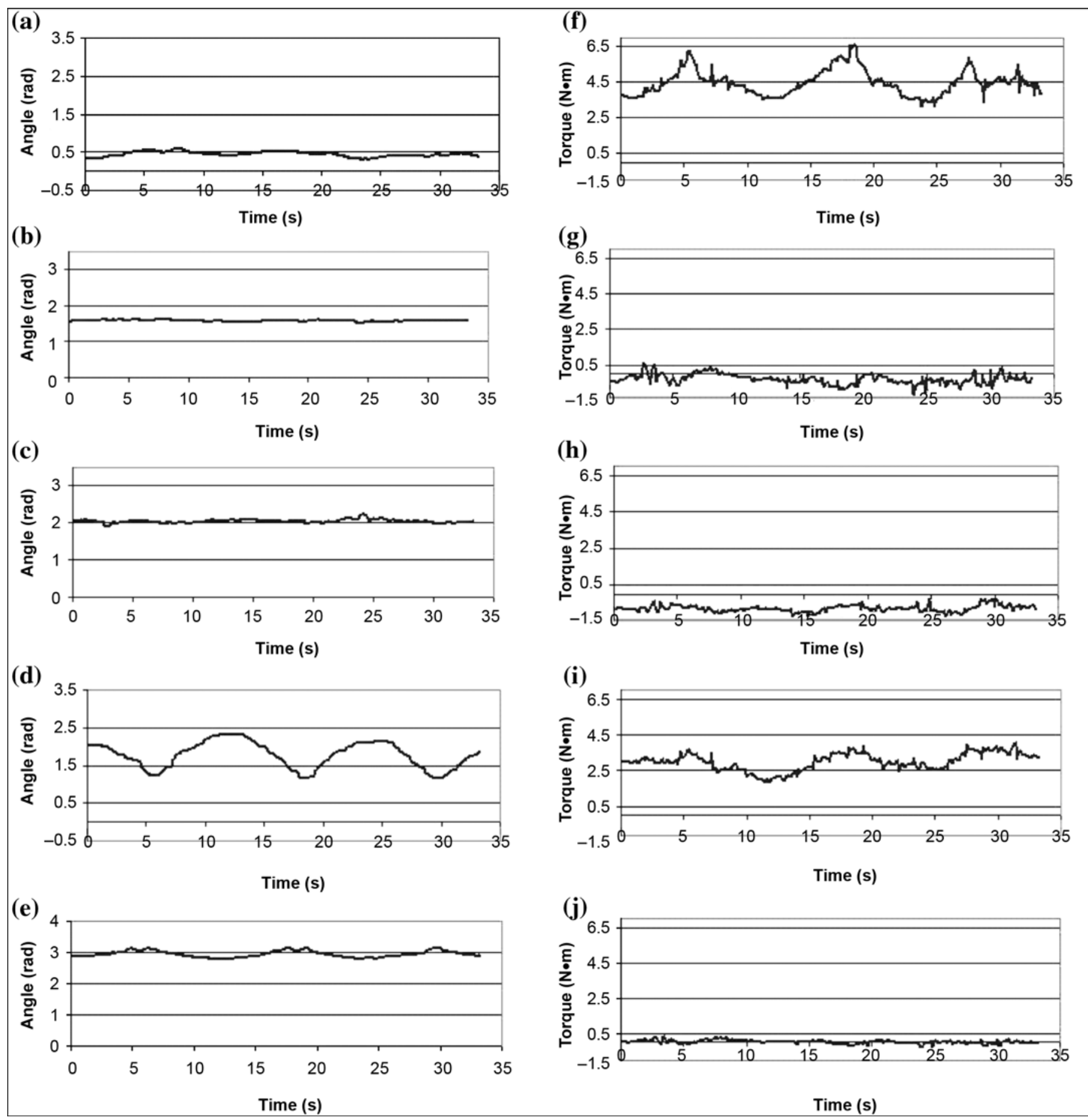

Figure 10.

Elbow exercise results for (a)-(e) measured angle for modeled joint degrees of freedom (DOF) 1-5, respectively, and (f)-(j) modeled torque for modeled joint DOF 1-5, respectively. Joint DOF designations are as follows: 1 represents shoulder flexion/extension, 2 represents shoulder abduction/ adduction, 3 represents shoulder internal/external rotation, 4 represents elbow flexion/extension, and 5 represents elbow pronation/supination.

The results from the combined shoulder and elbow exercise are illustrated in Figure 12. A comparison between the exercise trajectory and the modeled joints' displacement and torque confirmed the nature of the combined exercise and that the model was capable of accommodating such a complicated 3-D therapy exercise. 
(a)

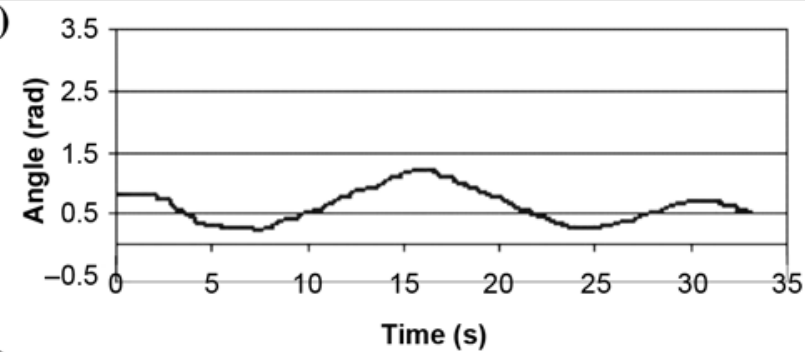

(b)

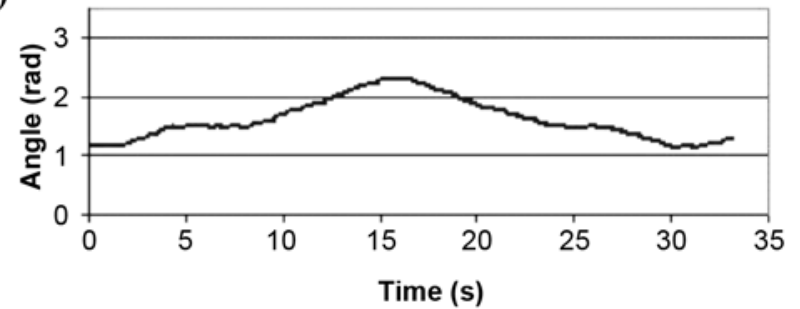

(c)

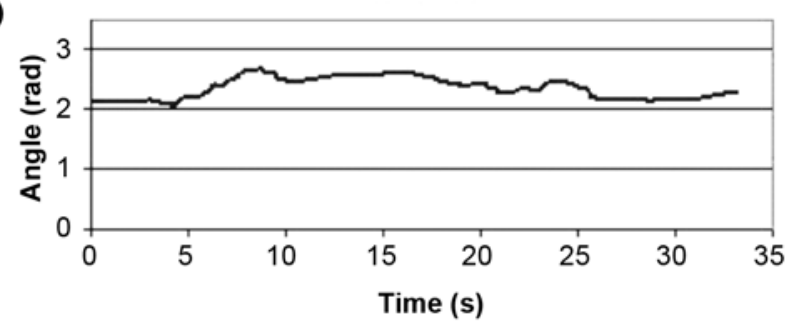

(d)

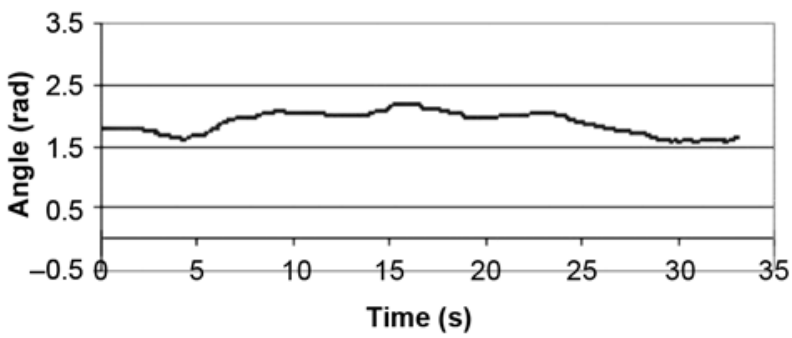

(e)

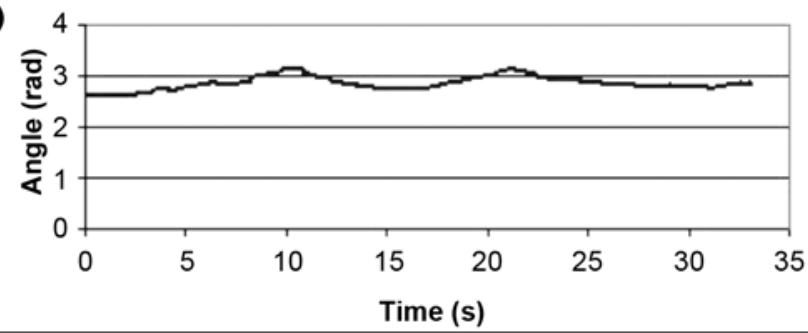

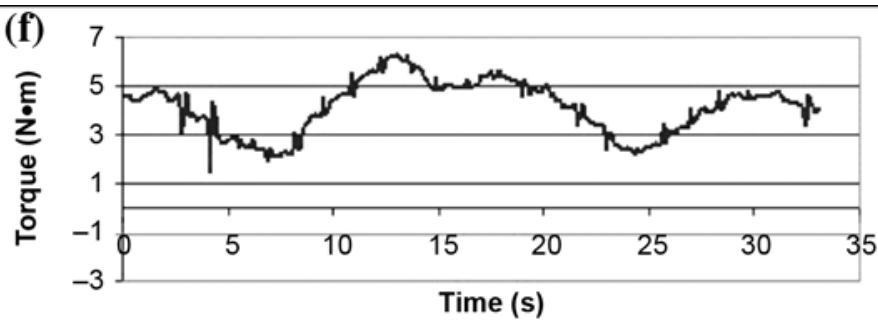

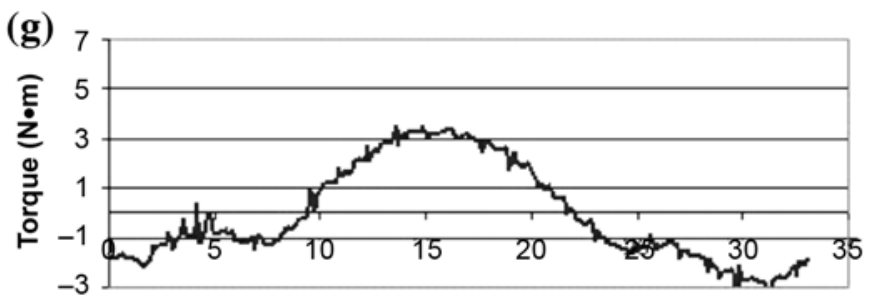

Time (s)

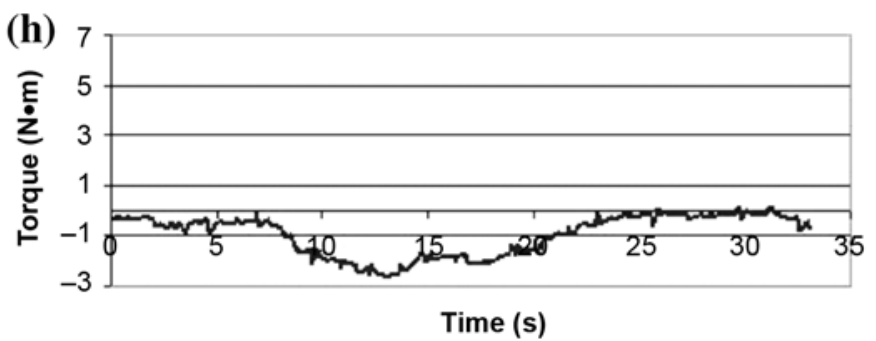

(i)

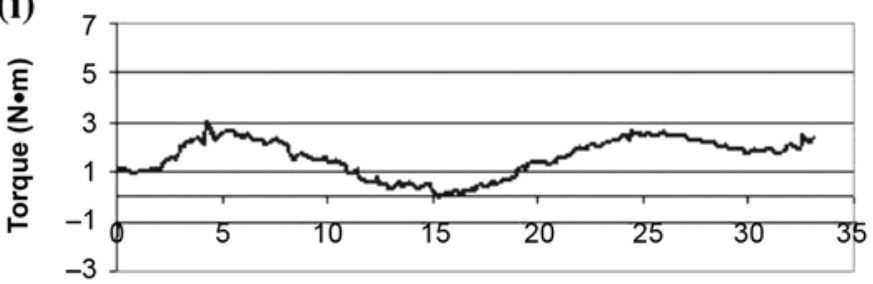

Time (s)

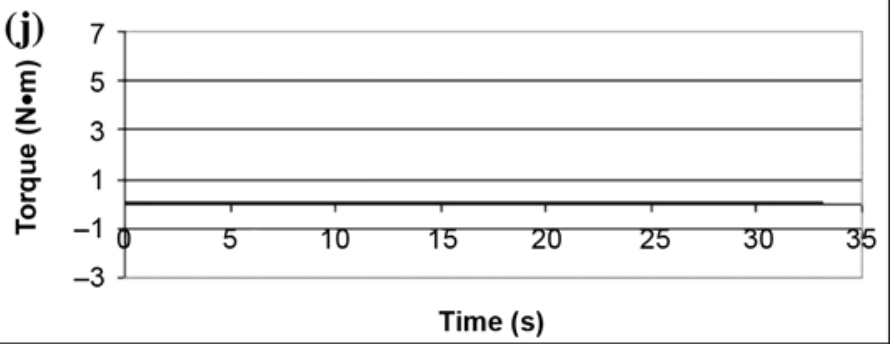

Figure 11.

Shoulder exercise results for (a)-(e) measured angle for modeled joint degrees of freedom (DOF) 1-5, respectively, and (f)-(j) modeled torque for modeled joint DOF 1-5, respectively. Joint DOF designations are as follows: 1 represents shoulder flexion/extension, 2 represents shoulder abduction/ adduction, 3 represents shoulder internal/external rotation, 4 represents elbow flexion/extension, and 5 represents elbow pronation/supination.

We can conclude from Figures 10-12 that the combined exercise was the most complicated exercise. Therefore, it generated more torque and involved more DOF. Analysis of the shoulder-exercise data indicated that the cross-body motion of the UL, with the change in elevation, generated a high reaction force from gravity when the UL was fully extended and parallel to the ground. The subject's UL was kept straight throughout the shoulder 
(a)

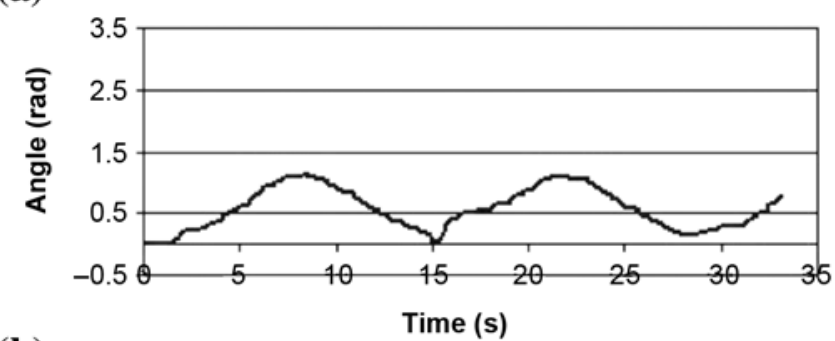

(b)

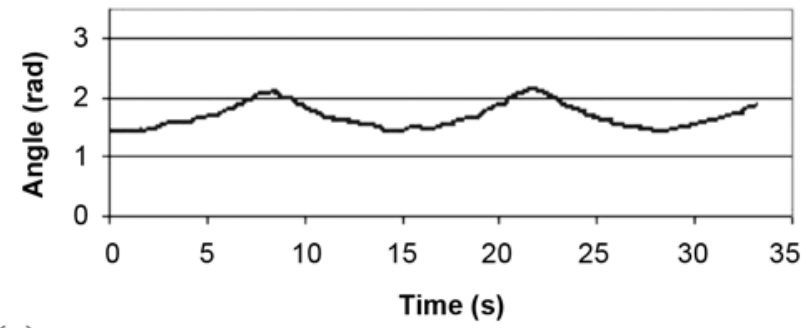

(c)

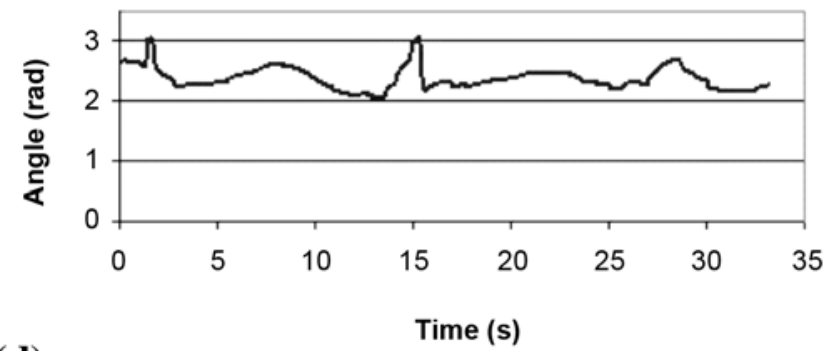

(d)

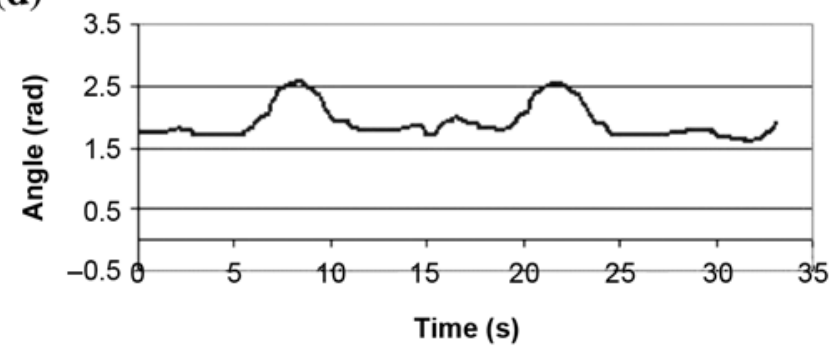

(e)

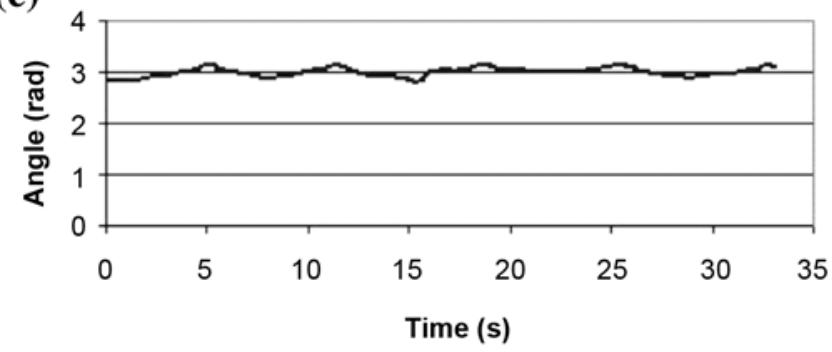

(f)

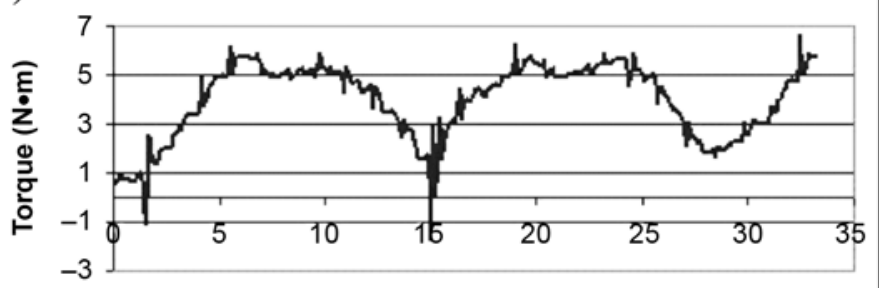

(g)

Time (s)

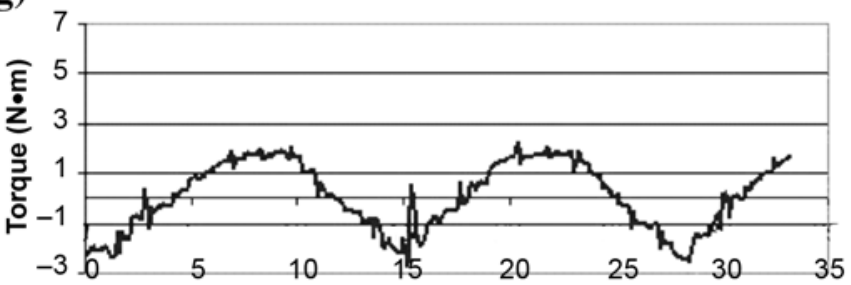

(h)

Time (s)

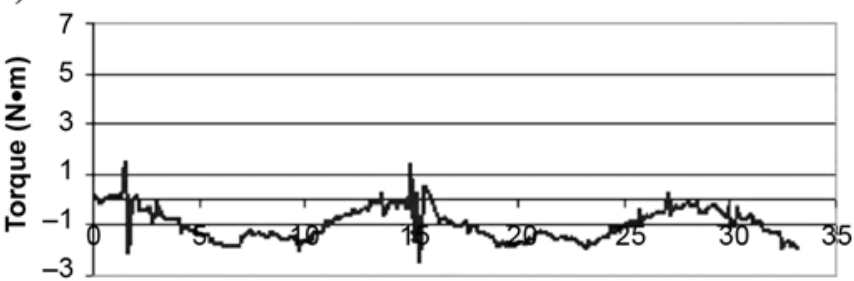

(i)

Time (s)

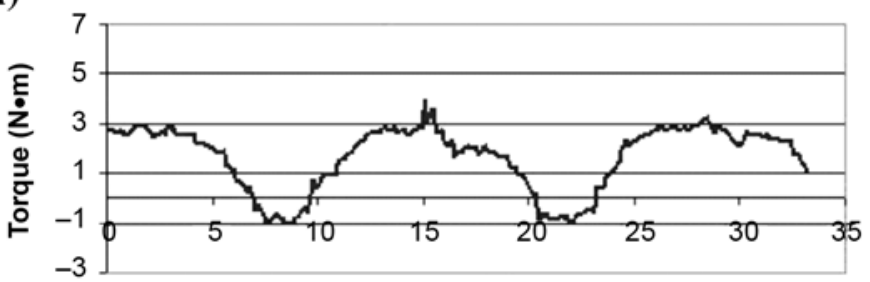

Time (s)

(j)

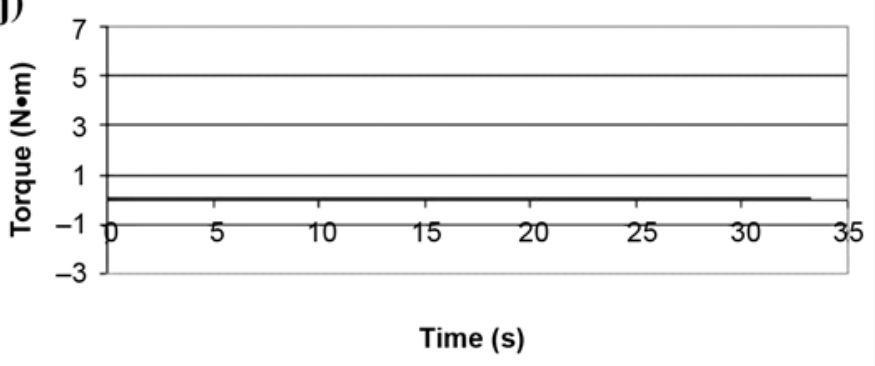

Figure 12.

Combined exercise results for (a)-(e) measured angle for modeled joint degrees of freedom (DOF) 1-5, respectively, and (f)-(j) modeled torque for modeled joint DOF 1-5, respectively. Joint DOF designations are as follows: 1 represents shoulder flexion/extension, 2 represents shoulder abduction/adduction, 3 represents shoulder internal/external rotation, 4 represents elbow flexion/extension, and 5 represents elbow pronation/ supination. 
exercise, which indeed strained the elbow and shoulder articulation at joint DOF 4, 2, and 1 as can be clearly seen from the experimental data of the model performance. The data presented in these figures have been used to experimentally verify the force and torque at the UL's joints generated by the therapy exercise.

The precision of the exercises was found to be near exact when subjects were removed from the system and then reattached. This process involved removing the 3-D pose sensors from the subject's UL and disconnecting the subject from the custom splint attached to the force sensor. Figure 13 shows the recorded 3-D trajectory for the same elbow exercise performed by the same subject in different sessions (1 and 2) at different times. The maximum difference between the wrist locations in the two sessions was only $5 \mathrm{~mm}$ and the average difference only $1 \mathrm{~mm}$. This level of precision is extremely useful when isolating the differences in joint torque from one session to another, because it ensures that the change is not related to a difference in the exercise. This result proves the repeatability in teaching, learning, sensing, and task performance of the integrated systems and indicates the capability of the model to recreate the same trajectory of the UL at different times. The only variable affecting the output is the difference in subject performance from session to session. Figure 13 shows $x, y$, and $z$ coordinates of the wrist in two separate exercises sessions.

After multiple repetitions, subjects tended to learn the exercise and their resistance to the robotic movement reduced. This trend in the force data showed a decrease in force along all axes over time (Figure 14). This force feedback may provide some understanding of the subject's recovery (normality of movement for passive mode therapy) and could be used as a measure of the exercise regime's effectiveness. This measurement could be achieved if a correlation could be found between the force feedback before training, during training, and at the end of the training program.

The velocity profiles of four subjects (during shoulder exercise) are presented in Figure 15. We can conclude that the exercise velocity profiles recorded by the model were similar when the wrist was moved at a relatively constant speed during each repetition of the exercise and stopped between each repetition.

A comparison between the trajectory of the shoulder exercise taught to the robot by the therapist and the trajectory achieved by three subjects with the help of the therapeutic robot is presented in Figure 16. The three subjects'

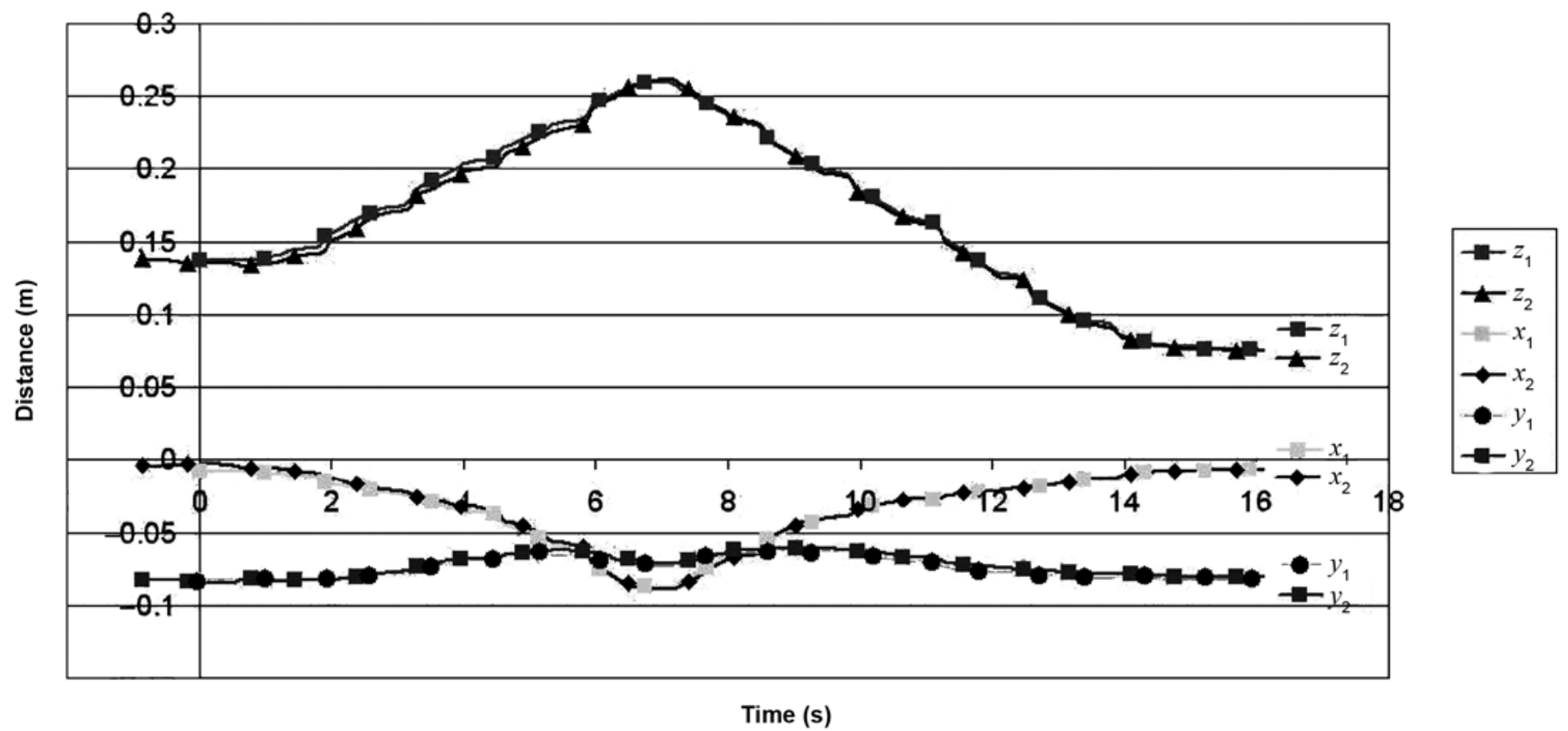

Figure 13.

Repeatability test of system for learned exercise. Recorded 3-dimensional trajectory ( $x, y$, and $z$ coordinates) for same elbow exercise performed by same subject in different sessions at different times. $1=\operatorname{session} 1,2=\operatorname{session} 2$. 


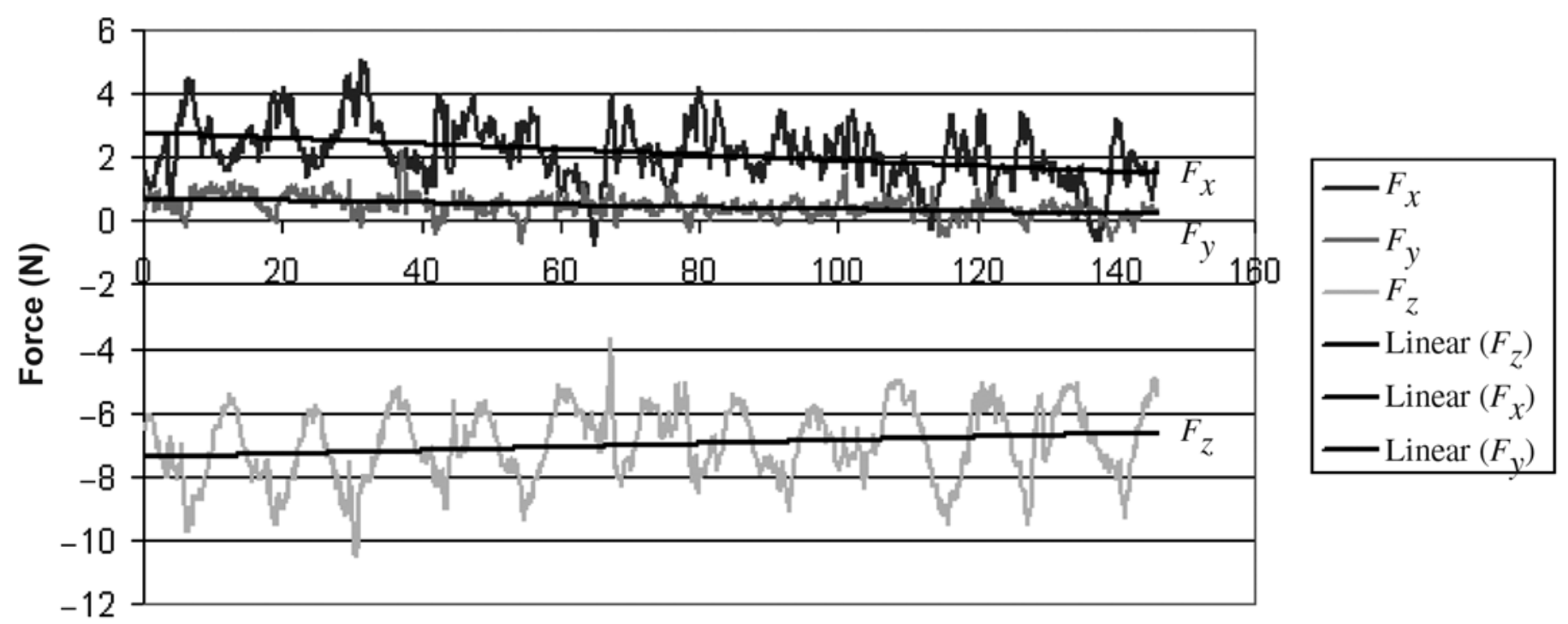

Time (s)

Figure 14.

Subject progress through shoulder therapy exercise; force trends at wrist over multiple repetitions. $F_{x}=$ force along $x$-axis, $F_{y}=$ force along $y$-axis, $F_{z}=$ force along $z$-axis.
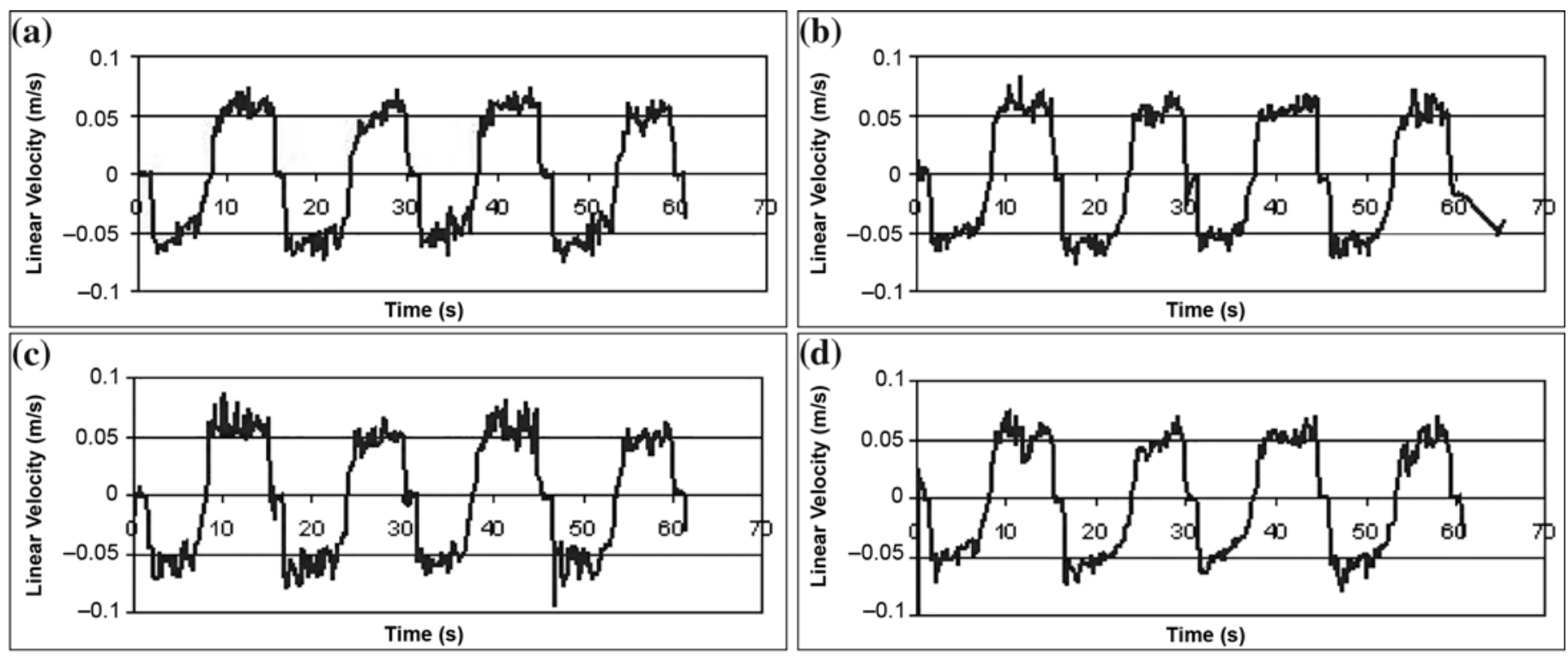

Figure 15.

Velocity changes over time for 4 subjects' wrists over course of 4 repetitions of shoulder exercise. Wrist velocity along z-axis for (a) subject A, (b) subject B, (c) subject C, and (d) subject D.

weight and dimensions are presented in Table 4. Only three subjects' trajectory data are presented because the other subjects' trajectories simply overlapped and cluttered the figure, without adding any further information. The exercises were consistent between different subjects; the largest deviation was only $4.5 \mathrm{~cm}$. This deviation was due to the method of connecting the subject to the robot during the preliminary trials, in which the patient was attached to the robot at the end of the hand and measurements were taken at the wrist. A portion of this difference was due to 
variation in hand size from one subject to another. This difference allowed the subject's wrist to move freely, which caused fluctuations in the output. The data presented show that the robotic system has the adaptability, repeatability, and reproducibility capabilities for performing the same exercise for different subjects at different times. The data also confirm the accommodation of the system with the extent of intersubject variability. Future research is needed to verify the monitoring capability of the model when it is used for postroke UL therapy over an extended period of time.

\section{DISCUSSION AND CONCLUSIONS}

A dynamic biomechanical 3-D model was developed for assessing and monitoring the trajectory, position, orientation, force, and torque generated by UL movement during robot-assisted therapy. The model could be a useful tool for enhancing the functionality of poststroke robot-assisted UL therapy. The data from the model could help the therapist quantify the actual resistance force at each joint and the quality of the exercise trajectory achieved by the subject. A decrease in resistance force in the passive mode and/or a reduction in trajectory deviation in the active mode indicated that subject performance improved. Therefore, the clinician could move to implement an exercise of greater difficulty. On the other hand, if such a result was not recorded, the clinician could change the exercise or reduce its difficulty. The model may also help the clinician identify the muscle group used by the subject to perform a task by identifying the orientation of the limb and subsequent torque magnitudes at the joints. This capability could indicate whether a weak muscle improved or the subject was depending more on a stronger muscle to complete the exercise.

The individualized segment inertial parameters and masses, which are difficult to measure for each patient, were based on anthropometric data. The model simultaneously monitored real-time biofeedback measured by a 6-DOF force sensor and 3-D pose sensors. The model also computed the 5-DOF joint reaction forces and torques generated by the UL when the therapeutic robot manipulated it in a therapy exercise. We analyzed the basic ADL that the poststroke patient would need to perform independently and concluded that 3-DOF for the shoulder and 2-DOF for the elbow joint were the major DOF necessary for these activities.

That the position of the robot and the position reported by the 3-D pose sensors were similar was verified by setting the 3-D pose sensors in stationary positions within the robot's work space and recording a single-point exercise. When the exercise was performed, the robot moved to the location of the 3-D pose sensors in all tests. The sensors were not mounted directly to the robot because close proximity of the sensors to the metal of the robot would have negative effects on the sensor readings. Having shown that the robot reached the location of the sensor using the data collected from the sensor to create the location, we believe that the only offset from robot location to sensor location was because of the orientation of the patient's limb. The measured precision of the robot as it traveled a preprogrammed trajectory was $\pm 0.05 \mathrm{~mm}$.

The system's performance was evaluated based on the investigation conducted with 20 nondisabled human subjects. The model's results confirmed the nature of the

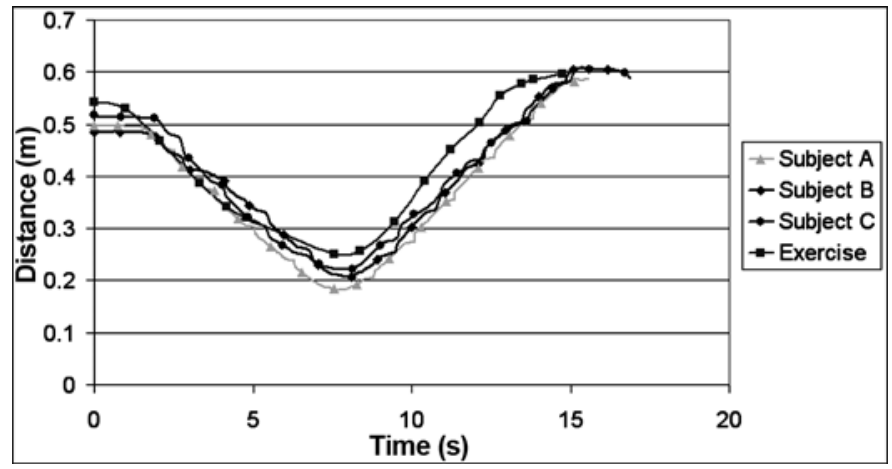

Figure 16.

Comparison of exercise taught to robot and location along $\mathrm{z}$-axis of subject's wrist during course of exercise for 3 subjects (A, B, and C).

Table 4.

Data of three subjects (A, B, and C) presented in Figure 16.

\begin{tabular}{cccccc}
\hline Subject & Sex/Age (yr) & Forearm Length (cm) & Upper-Arm Length (cm) & Height (cm) & Weight (kg) \\
\hline A & Male/24 & 38 & 26 & 178 \\
B & Female/24 & 33 & 28 & 168 & 82 \\
C & Male/44 & 42 & 29 & 175 & 81 \\
\hline \hline
\end{tabular}


three therapy exercises (elbow, shoulder, and combined) in the experimental protocol. Results also showed good repeatability of the exercises when subjects were removed from the system and reattached to perform the same exercise at a different time. The robotic system's adaptability, repeatability, and reproducibility capabilities have been verified for the same exercise for the same subject or different subjects at different times.

\section{ACKNOWLEDGMENTS}

We would like to thank Patti Galvin, MD, and Ranjit Singh, MD (Guelph, Ontario, Canada), for their valuable discussions and feedback.

This material is the result of work supported with resources from the Natural Science and Engineering Research Council of Canada.

The authors have declared that no competing interests exist.

\section{REFERENCES}

1. Feeney DM, Gonzalez A, Law WA. Amphetamine, haloperidol, and experience interact to affect rate of recovery after motor cortex injury. Science. 1982;217(4562):855-57. [PMID: 7100929]

2. Butefisch C, Hummelsheim H, Denzler P, Mauritz KH. Repetitive training of isolated movement improves the outcome of motor rehabilitation of the centrally paretic hand. J Neurol Sci. 1995;130(1):59-68. [PMID: 7650532]

3. Reinkensmeyer DJ, Kahn LE, Averbuch M, McKenna-Cole A, Schmit BD, Rymer WZ. Understanding and treating arm movement impairment after chronic brain injury: Progress with the ARM guide. J Rehabil Res Dev. 2000;37(6):653-62. [PMID: 11321001]

4. Reinkensmeyer DJ, Emken JL, Cramer SC. Robotics, motor learning, and neurologic recovery. Annu Rev Biomed Eng. 2004;6:497-525. [PMID: 15255778]

5. Keramas LG. Robot technology fundamentals. Florence (KY): Delmar Publishers; 1999.

6. Erlandson RF. Applications of robotic/mechatronic systems in special education, rehabilitation therapy, and vocational training: A paradigm shift. IEEE Trans Rehabil Eng. 1995;3(1):22-34.

7. Dijkers MP, DeBear PC, Erlandson RF, Kristy K, Geer DM, Nicholes A. Patient and staff acceptance of robot technology in occupational therapy: A pilot study. J Rehabil Res Dev. 1991;28(2):33-44. [PMID: 2066869]
8. Austin M, Cozens J, Plummer A, Seedhom B. Controller problems in robotic therapy for upper limb rehabilitation: An initial study. In: Proceedings of the 21st Annual RESNA Conference; 1998 Jun 26-30; Minneapolis, Minnesota. Arlington (VA): RESNA; 1998. p. 292-304.

9. Cozens JA. Robotic assistance of an active upper limb exercise in neurologically impaired patients. IEEE Trans Rehabil Eng. 1999;7(2):254-56.

10. Hogan N, Krebs HI, Charnnarong J, Srikrisha P, Sharon A. MIT-MANUS: A workstation for manual therapy and training II. In: Das H, editor. International Society of Optical Engineering (SPIE) Telemanipulator Technology Proceedings; 1992 Nov 15-20; Boston, Massachusetts. Bellingham (WA): SPIE. Mar 1993. p. 28-34.

11. Krebs HI, Palazzolo JJ, Dipietro L, Ferraro M, Krol J, Rannekleiv K, Volpe BT, Hogan N. Rehabilitation robotics: Performance-based progressive robot-assisted therapy. Auton Robots. 2003;15(1):7-20.

12. Burgar CG, Lum PS, Shor PC, Van der Loos HF. Development of robots for rehabilitation therapy: The Palo Alto VA/Stanford experience. J Rehabil Res Dev. 2000;37(6): 663-73. [PMID: 11321002]

13. Khan LE, Zygman ML, Rymer WZ, Reinkensmeyer DJ. Effect of robot-assisted and unassisted exercise on functional reaching in chronic hemiparsis. In: Proceedings of the 23rd Annual IEEE EMBS International Conference; 2001 Oct 23-28; Istanbul, Turkey. Piscataway (NJ): IEEE. p. 1344-47.

14. Rab G, Petuskey K, Bagley A. A method for determination of upper extremity kinematics. Gait Posture. 2002;15(2): 113-19. [PMID: 11869904]

15. Fritz M. An improved biomechanical model for simulating the strain of the hand-arm system under vibration stress. J Biomech. 1991;24(12):1165-71. [PMID: 1769981]

16. Raikova R. A general approach for modelling and mathematical investigation of the human upper limb. J Biomech. 1992;25(8):857-67. [PMID: 1639830]

17. Seireg A, Arvikar RJ. A mathematical model for evaluation of forces in lower extremities of the musculo-skeletal system. J Biomech. 1973;6(3):313-26. [PMID: 4706941]

18. Zajac FE, Gordon ME. Determining muscle's force and action in multi-articular movement. Exerc Sport Sci Rev. 1989;17:187-230. [PMID: 2676547]

19. Riener R, Straube A. Inverse dynamics as a tool for motion analysis: Arm tracking movements in cerebellar patients. J Neurosci Methods. 1997;72(1):87-96. [PMID: 9128172$]$

20. Tognetti A, Lorussi F, Bartalesi R, Quaglini S, Tesconi M, Zupone G, De Rossi D. Wearable kinesthetic system for capturing and classifying upper limb gesture in post-stroke rehabilitation. J Neuroengineering Rehabil. 2005;2(1):8.

[PMID: 15743530] 
21. Murray IA, Johnson GR. A study of the external forces and moments at the shoulder and elbow while performing every day tasks. Clin Biomech (Bristol, Avon). 2005;19(6):586-94. [PMID: 15234482]

22. Romilly DP, Anglin C, Gosine RG, Hershler C, Raschke SU. A functional task analysis and motion simulation for the development of a powered upper-limb orthosis. IEEE Trans Rehabil Eng. 1994;2(3):119-29.

23. Winter DA. Biomechanics and motor control of human movement. New York (NY): John Wiley \& Sons; 1990.

24. Van der Helm FC. Analysis of the kinematic and dynamic behavior of the shoulder mechanism. J Biomech. 1994;27(5): 527-50. [PMID: 8027089]

25. Hogfors C, Peterson B, Sigholm G, Herberts P. Biomechanical model of the human shoulder joint-II. The shoulder rhythm. J Biomech. 1991;24(8):699-709. [PMID: 1918093]

26. Karlsson D, Peterson B. Towards a model for force predictions in the human shoulder. J Biomech. 1992;25(2):189-99. [PMID: 1733994]

27. Nieminen H, Niemi J, Takala EP, Viikari-Juntura E. Loadsharing patterns in the shoulder during isometric flexion tasks. J Biomech. 1995;28(5):555-66. [PMID: 7775491]

28. Khalili D, Zomlefer M. An intelligent robotic system for rehabilitation of joints and estimation of body segment parameters. IEEE Trans Biomed Eng. 1988;35(2):138-46. [PMID: 3350539]

29. Rittenhouse DM, Abdullah HA, Runciman RJ, Basir OA. A neural network model for reconstructing EMG signals from eight shoulder muscles: Consequences for rehabilitation robotics and biofeedback. J Biomech. 2006;39(10): 1924-32. [PMID: 15993412]

30. Craig JJ. Introduction to robotics: Mechanics and control. 3rd ed. Reading (MA): Prentice Hall; 2003.

31. Denavit J, Hartenberg RS. A kinematic notation for lower pair mechanisms based on matrices. J Applied Mech. 1955; 77:215-21.

32. Drillis R, Contimi R. Body segment parameters. School of Eng Sci Tech Rep. No. 1166-1203. New York (NY): New York University; 1966.

33. Dempster WT. Space requirements of the seated operator: Geometrical, kinematic, and mechanical aspects of the body with special references to the limbs. Wright-Patterson Air Force Base (OH): Wright Air Development Center; 1955.

Submitted for publication March 8, 2006. Accepted in revised form November 29, 2006. 